\title{
Spectroscopic Characterization of Nicotinoyl and Isonicotinoyl Nitrenes \\ and the Photointerconversion of 4-Pyridylnitrene with
}

\section{Diazacycloheptatetraene}

\author{
Qian Liu, ${ }^{\dagger}$ Yuanyuan Qin,${ }^{\dagger}$ Yan Lu,${ }^{\dagger}$ Curt Wentrup,,$\stackrel{*}{\dagger}$ Xiaoqing Zeng ${ }^{*}, \dagger$ \\ ${ }^{\dagger}$ College of Chemistry, Chemical Engineering and Materials Science, Soochow University, 215123 Suzhou, \\ China \\ E-mail: xqzeng@suda.edu.cn
}

${ }^{\sharp}$ School of Chemistry and Molecular Biosciences, The University of Queensland, Brisbane, Queensland 4072, Australia

E-mail: wentrup@uq.edu.au

Table of Contents

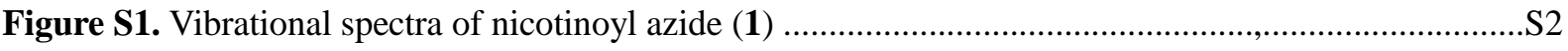

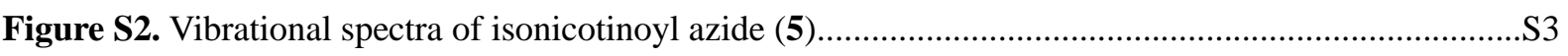

Figure S3. $\mathrm{N}_{2}$-matrix IR spectra showing the photodecomposition of nicotinoyl azide (1)......................S4

Figure S4. $\mathrm{N}_{2}$-matrix IR spectra showing the photodecomposition of isonicotinoyl azide (5)..................S5

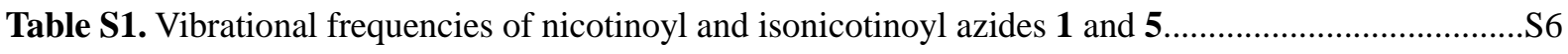

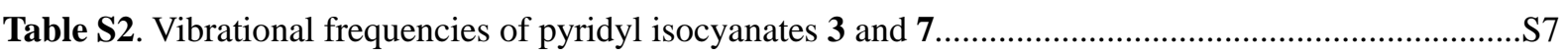

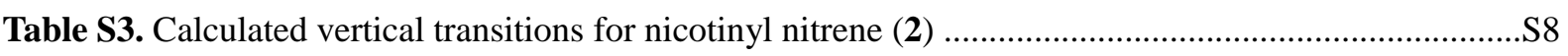

Table S4. Calculated vertical transitions for isonicotiny nitrene $(6)$ and 4-pyridyl nitrene (8)..................S8

Calculated atomic coordinates (in Angstrom) and energies (in Hartree) for all optimized structures..........S9 


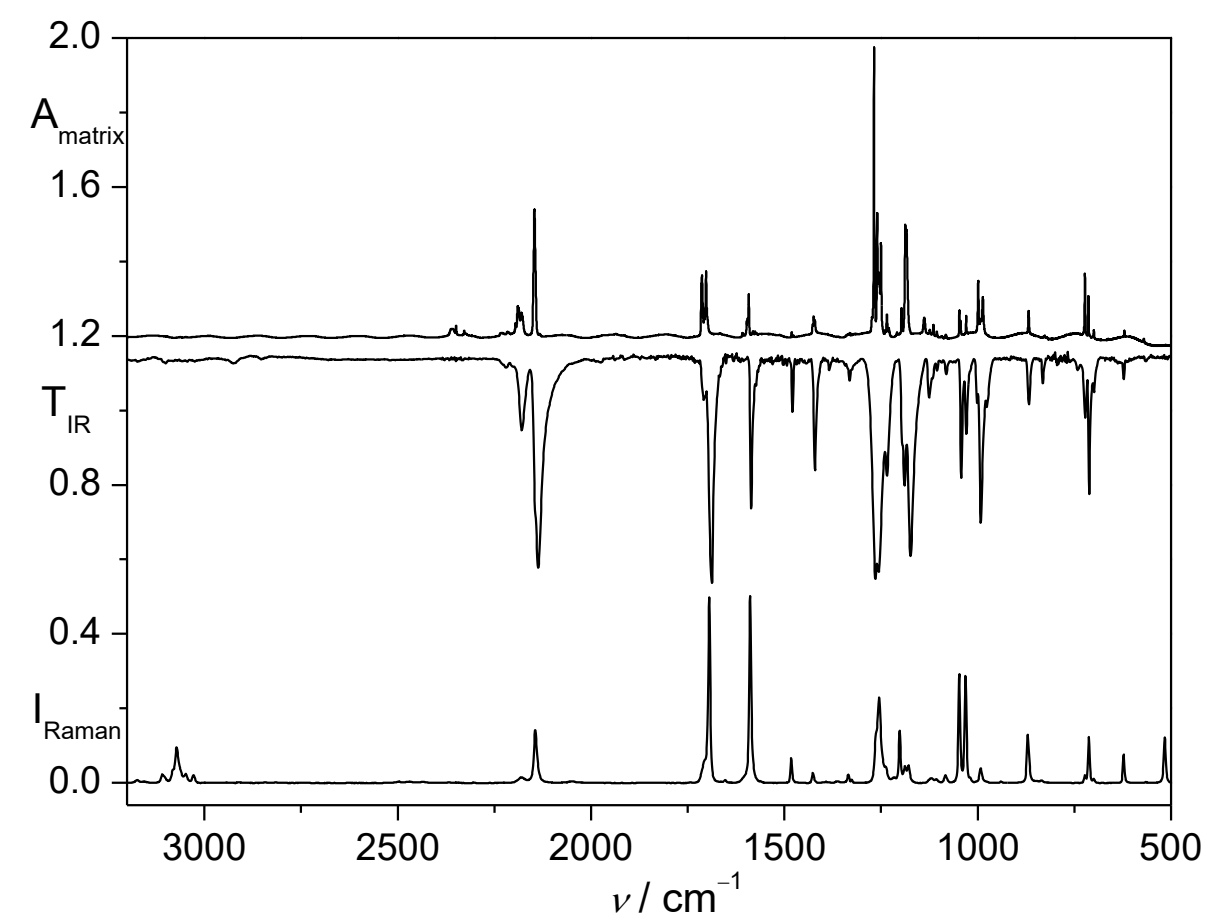

Figure S1. Upper trace: IR spectrum of nicotinoyl azide (1) isolated in $\mathrm{N}_{2}$-matrix at $15 \mathrm{~K}$ (absorbance $A$, resolution: $0.5 \mathrm{~cm}^{-1}$ ). Middle trace: IR spectrum of solid nicotinoyl azide at $298 \mathrm{~K}$ (transmission $T$, resolution: $2 \mathrm{~cm}^{-1}$ ). Lower trace: Raman spectrum of solid nicotinoyl azide at $298 \mathrm{~K}$ (Raman intensity $I$, resolution: $2 \mathrm{~cm}^{-1}$ ). 


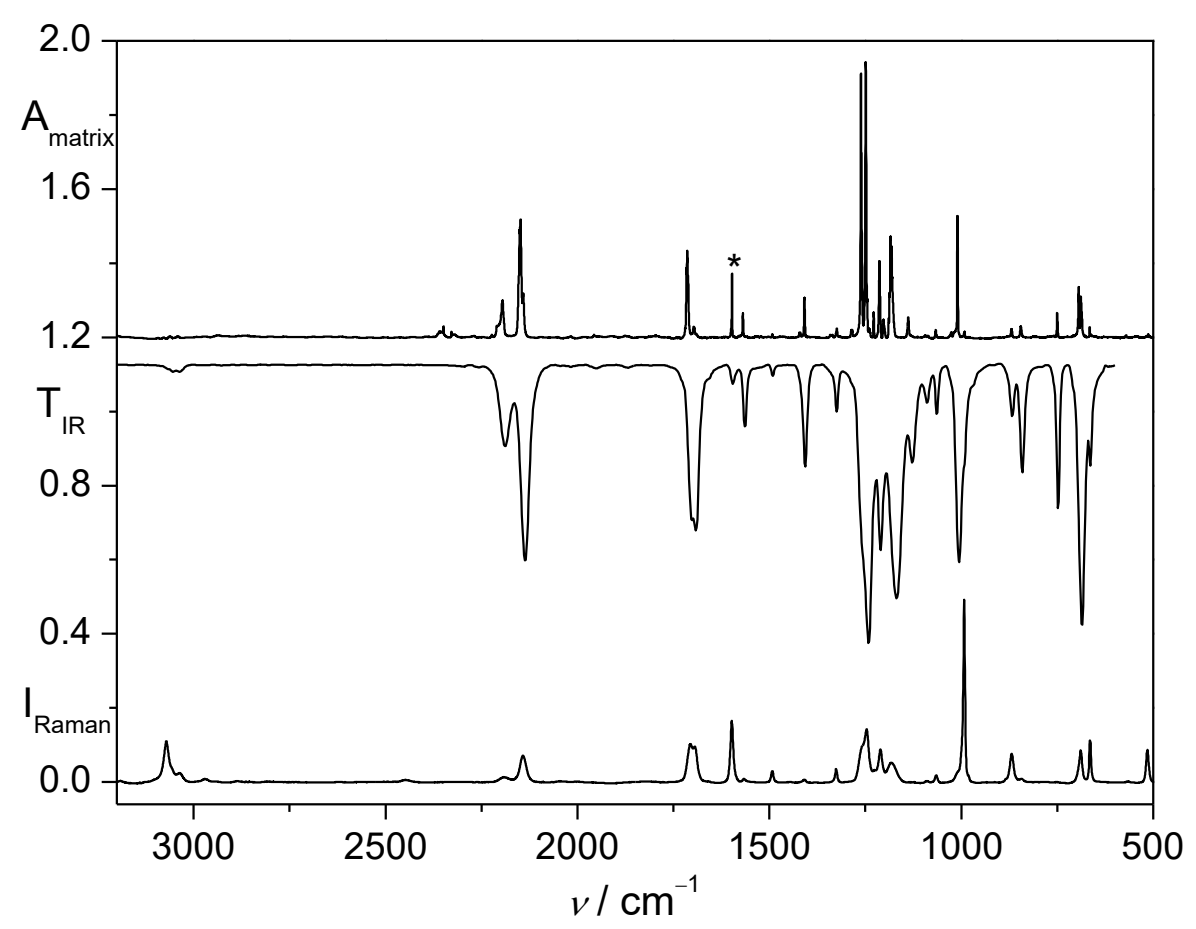

Figure S2. Upper trace: IR spectrum of isonicotinoyl azide (5) isolated in $\mathrm{N}_{2}$-matrix at $15 \mathrm{~K}$ (absorbance $A$, resolution: $0.5 \mathrm{~cm}^{-1}$ ). Middle trace: IR spectrum of liquid isonicotinoyl azide at $298 \mathrm{~K}$ (transmission $T$, resolution: $2 \mathrm{~cm}^{-1}$ ). Lower trace: Raman spectrum of liquid isonicotinoyl azide at $298 \mathrm{~K}$ (Raman intensity $I$, resolution: $2 \mathrm{~cm}^{-1}$ ). Band associated with $\mathrm{H}_{2} \mathrm{O}$ is marked with an asterisk. 


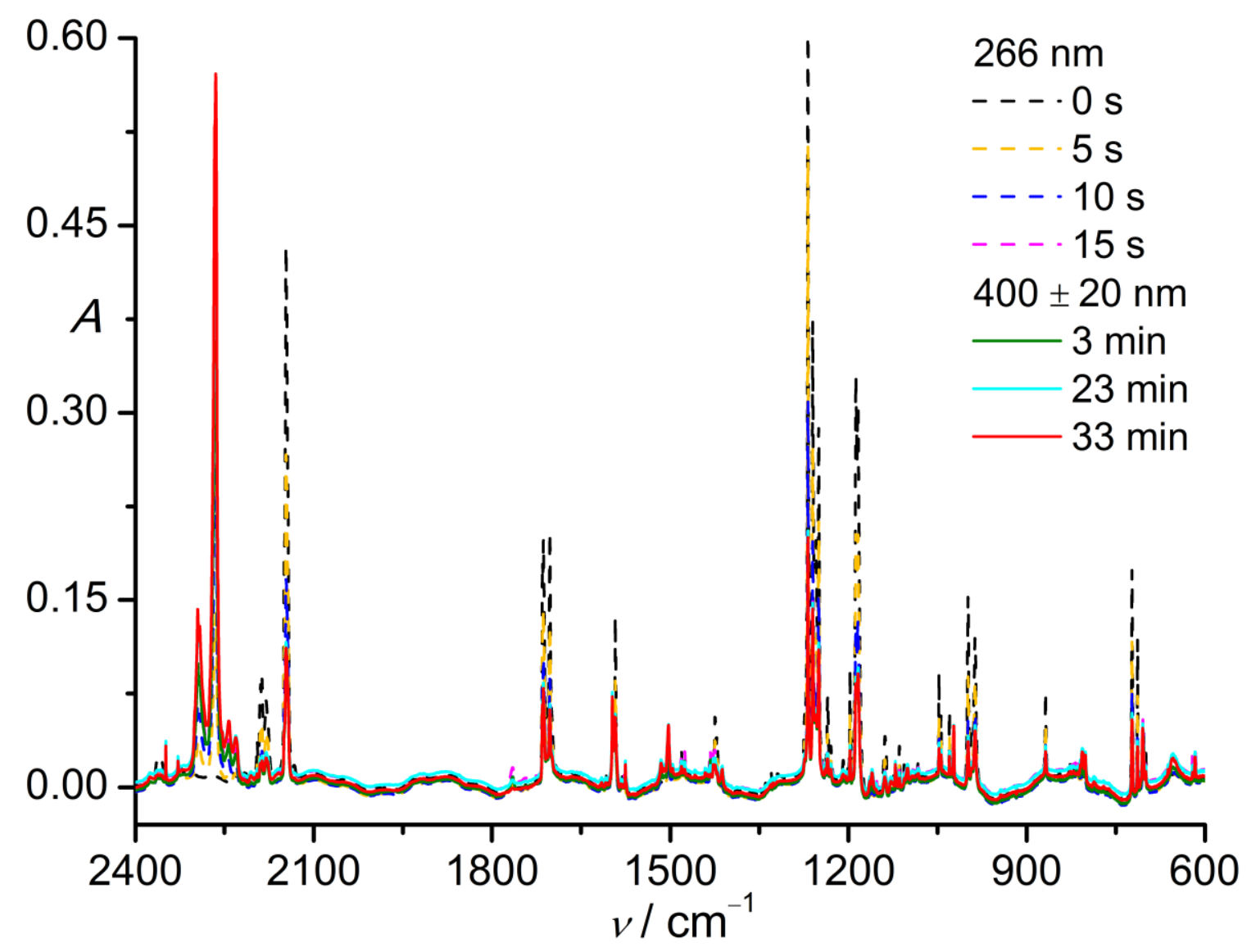

Figure S3. $\mathrm{N}_{2}$-matrix IR spectra showing the photodecomposition of nicotinoyl azide (1) at $15 \mathrm{~K}$. 


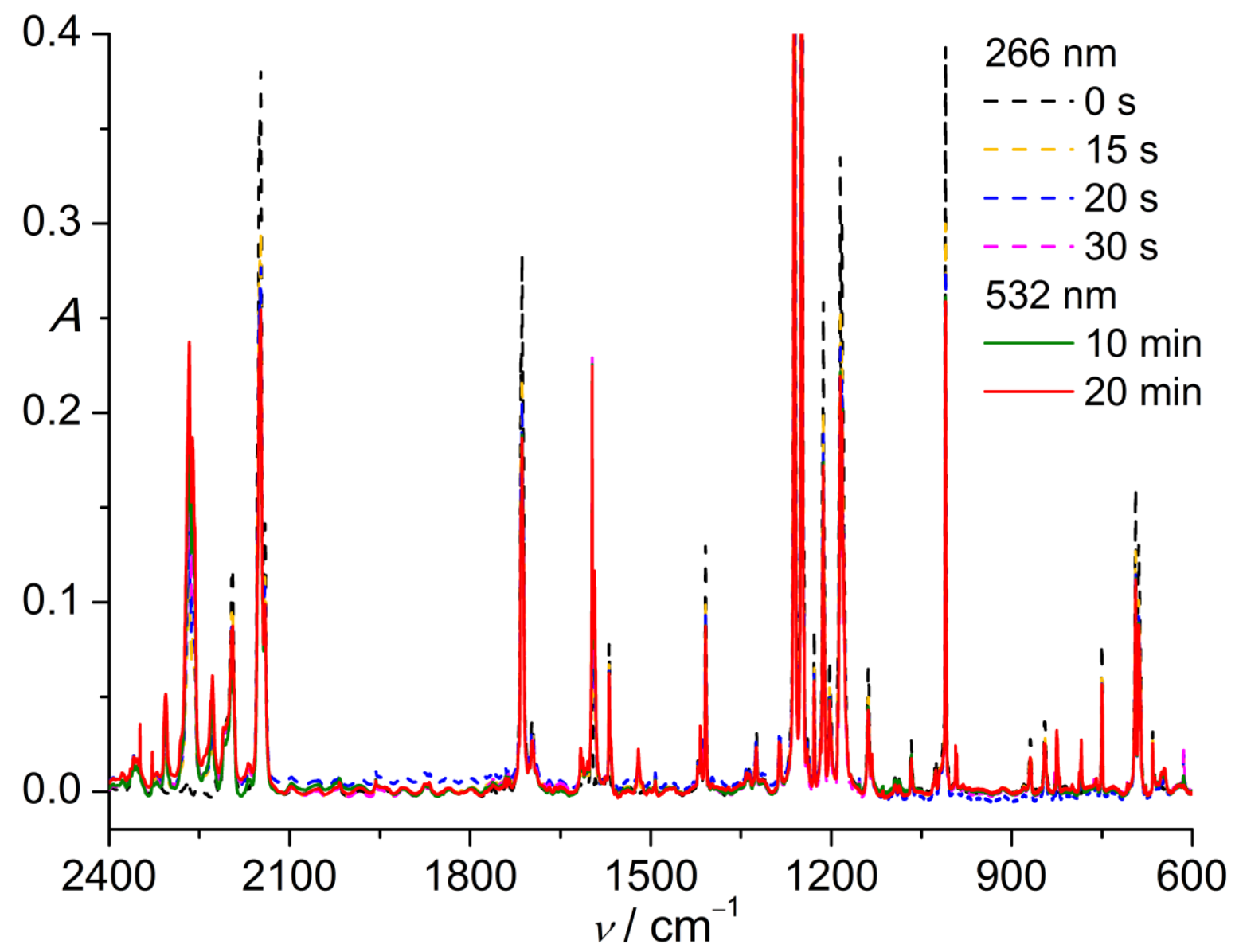

Figure S4. $\mathrm{N}_{2}$-matrix IR spectra showing the photodecomposition of isonicotinoyl azide (5) at $15 \mathrm{~K}$. 
Table S1. Experimentally observed and calculated vibrational frequencies $\left(>500 \mathrm{~cm}^{-1}\right)$ of nicotinoyl azide (1) and isonicotinoyl azide (5).

\begin{tabular}{|c|c|c|c|c|c|c|c|c|}
\hline \multirow{2}{*}{\multicolumn{3}{|c|}{$\begin{array}{l}\text { Nicotinoyl azide (1) } \\
\text { Observed }^{\mathrm{a}}\end{array}$}} & \multirow[b]{2}{*}{$\begin{array}{l}\text { Calculated }\left(I_{\mathrm{IR}}\right)\left[I_{\mathrm{Ra}}\right]^{\mathrm{b}} \\
\text { anti-syn }\end{array}$} & \multicolumn{4}{|c|}{ Isonicotinoyl azide (5) } & \multirow[t]{2}{*}{ Assignment ${ }^{\mathrm{c}}$} \\
\hline & & Raman (solid) & & $\begin{array}{l}\text { Observed }^{\mathrm{a}} \\
\text { IR (liquid) }\end{array}$ & IR (matrix) & Raman (liquid) & $\begin{array}{l}\text { Calculated }\left(I_{\mathrm{IR}}\right)\left[I_{\mathrm{Ra}}\right]^{\mathrm{b}} \\
\text { syn }\end{array}$ & \\
\hline \multirow[t]{4}{*}{$3099 \mathrm{vw}$} & $3096.7 \mathrm{vw}$ & $3109 \mathrm{vw}$ & $3107(5)[135]$ & & $3093.0 \mathrm{vw}$ & & $3114(<1)[87]$ & $v_{\mathrm{s}}(\mathrm{CH})$ \\
\hline & $3069.1 \mathrm{vw}$ & $3072 \mathrm{~m}$ & $3091(4)[146]$ & & $3078.6 \mathrm{vw}$ & & $3110(3)[86]$ & $v_{\mathrm{as}}(\mathrm{CH})$ \\
\hline & $3061.2 \mathrm{vw}$ & & $3087(6)[34]$ & & $3061.1 \mathrm{vw}$ & $3071 \mathrm{~s}$ & $3064(6)[142]$ & $v_{\mathrm{as}}(\mathrm{CH})$ \\
\hline & $3048.4 \mathrm{vw}$ & 3027 vw & 3059 (13)[128] & $3054 \mathrm{vw}$ & $3040.7 \mathrm{vw}$ & $3036 \mathrm{w}$ & $3061(25)[110]$ & $v_{\mathrm{as}}(\mathrm{CH})$ \\
\hline $2137 \mathrm{vs}$ & 2146.7 vs & $2145 \mathrm{~s}$ & 2209 (646)[208] & $2136 \mathrm{~s}$ & 2149.4 vs & $2142 \mathrm{~s}$ & $2210(619)[196]$ & $v_{\mathrm{as}}\left(\mathrm{N}_{3}\right)$ \\
\hline $1688 \mathrm{vs}$ & $1713.1 \mathrm{~s}$ & $1694 \mathrm{vs}$ & $1692(237)[96]$ & $1692 \mathrm{~s}$ & $1714.0 \mathrm{~s}$ & $1694 \mathrm{~s}$ & $1698(202)[71]$ & $v(\mathrm{C}=\mathrm{O})$ \\
\hline \multirow[t]{2}{*}{$1586 \mathrm{~s}$} & $1592.1 \mathrm{~m}$ & 1588 vs & $1577(79)[145]$ & $1595 \mathrm{vw}$ & & $1598 \mathrm{~m}$ & $1578(3)[82]$ & $v(\mathrm{CC})$ \\
\hline & $1580.9 \mathrm{vw}$ & & $1554(10)[3]$ & $1564 \mathrm{w}$ & $1569.2 \mathrm{w}$ & $1566 \mathrm{vw}$ & $1549(24)[3]$ & $v(\mathrm{C}=\mathrm{C})$ \\
\hline $1479 \mathrm{w}$ & $1481.0 \mathrm{vw}$ & $1482 \mathrm{w}$ & $1462(3)[10]$ & $1491 \mathrm{vw}$ & $1492.5 \mathrm{vw}$ & $1492 \mathrm{w}$ & $1474(3)[7]$ & $\delta(\mathrm{CH})$ \\
\hline $1421 \mathrm{~m}$ & $1424.5 \mathrm{w}$ & $1427 \mathrm{vw}$ & $1406(35)[6]$ & $1407 \mathrm{~m}$ & $1408.9 \mathrm{~m}$ & $1409 \mathrm{vw}$ & $1397(28)[3]$ & $v(\mathrm{C}=\mathrm{C})$ \\
\hline \multirow[t]{2}{*}{$1331 \mathrm{vw}$} & $1330.3 \mathrm{vw}$ & $1335 \mathrm{vw}$ & $1320(2)[2]$ & $1325 \mathrm{w}$ & $1324.3 \mathrm{vw}$ & $1326 \mathrm{w}$ & $1312(5)[6]$ & $\delta(\mathrm{CH})$ \\
\hline & $1273.6 \mathrm{w}$ & & $1252(35)[13]$ & $1241 \mathrm{vs}$ & 1249.5 vs & $1246 \mathrm{~s}$ & $1244(766)[4]$ & $v\left(\mathrm{CN}_{\text {ring }}\right)$ \\
\hline $1260 \mathrm{vs}$ & $1268.2 \mathrm{vs}$ & $1255 \mathrm{~s}$ & $1247(839)[8]$ & $1210 \mathrm{~m}$ & $1213.3 \mathrm{~m}$ & $1211 \mathrm{w}$ & $1237(79)[17]$ & $v_{\mathrm{s}}\left(\mathrm{N}_{3}\right)$ \\
\hline $1235 \mathrm{~m}$ & $1235.1 \mathrm{w}$ & $1202 \mathrm{~m}$ & $1217(17)[71]$ & $1169 \mathrm{~s}$ & $1184.6 \mathrm{~s}$ & $1181 \mathrm{~m}$ & $1214(24)[55]$ & $v(\mathrm{CC})$ \\
\hline $1180 \mathrm{~m}$ & $1187.3 \mathrm{~s}$ & 1179 w & 1184 (11)[19] & $1128 \mathrm{~m}$ & $1138.4 \mathrm{w}$ & & $1195(38)[56]$ & $\delta(\mathrm{CH})$ \\
\hline $1124 \mathrm{w}$ & $1138.7 \mathrm{w}$ & $1118 \mathrm{vw}$ & $1100(29)[4]$ & $1089 \mathrm{w}$ & & $1090 \mathrm{vw}$ & 1073 (1)[2] & $\delta(\mathrm{CH})$ \\
\hline $1042 \mathrm{~s}$ & $1047.6 \mathrm{w}$ & $1048 \mathrm{~s}$ & $1035(29)[40]$ & $1064 \mathrm{w}$ & $1066.6 \mathrm{vw}$ & $1065 \mathrm{w}$ & $1057(7)[4]$ & Ring breathing \\
\hline \multirow[t]{2}{*}{$1029 \mathrm{~m}$} & $1029.9 \mathrm{w}$ & $1032 \mathrm{~s}$ & $1012(15)[26]$ & $1006 \mathrm{~s}$ & $1010.1 \mathrm{~s}$ & & $988(162)[7]$ & $\delta(\mathrm{CC})$ \\
\hline & & & $994(<1)[<1]$ & & & 993 vs & $985(<1)[<1]$ & $\gamma(\mathrm{CH})$ \\
\hline \multirow[t]{3}{*}{$992 \mathrm{~s}$} & $998.8 \mathrm{~m}$ & $992 \mathrm{w}$ & $976(174)[4]$ & & & & $981(3)[40]$ & $v\left(\mathrm{CN}_{\text {azide }}\right)$ \\
\hline & $986.6 \mathrm{~m}$ & & $972(<1)[<1]$ & & & & $972(<1)[<1]$ & $\gamma(\mathrm{CH})$ \\
\hline & & & $936(<1)[<1]$ & & & & $874(<1)[<1]$ & $\gamma(\mathrm{CH})$ \\
\hline 867 w & 868.4 w & $871 \mathrm{~m}$ & 843 (16)[8] & $867 w$ & $869.2 \mathrm{vw}$ & $869 \mathrm{~m}$ & 845 (8)[7] & $\delta\left(\mathrm{C}(\mathrm{O}) \mathrm{N}_{\text {azide }}\right)$ \\
\hline $832 \mathrm{vw}$ & $826.9 \mathrm{vw}$ & & $820(6)[<1]$ & $841 \mathrm{~m}$ & $845.2 \mathrm{vw}$ & & $840(13)[<1]$ & $\gamma(\mathrm{CH})$ \\
\hline $722 \mathrm{w}$ & $722.8 \mathrm{~m}$ & $722 \mathrm{vw}$ & $711(50)[<1]$ & $748 \mathrm{~m}$ & $750.2 \mathrm{w}$ & & $745(12)[<1]$ & $\gamma(\mathrm{CH})$ \\
\hline $712 \mathrm{~m}$ & $713.7 \mathrm{~m}$ & $713 \mathrm{~m}$ & 706 (27)[8] & & & & $689(36)[<1]$ & $\delta($ ring $)$ \\
\hline $699 \mathrm{vw}$ & 699.9 w & $699 \mathrm{vw}$ & $696(7)[<1]$ & $686 \mathrm{~s}$ & $689.0 \mathrm{~m}$ & $689 \mathrm{~m}$ & $680(56)[8]$ & $\gamma(\mathrm{CH})$ \\
\hline $622 \mathrm{vw}$ & $621.0 \mathrm{vW}$ & $623 \mathrm{w}$ & $614(3)[4]$ & $664 \mathrm{~m}$ & $665.6 \mathrm{vw}$ & $665 \mathrm{~m}$ & $660(4)[7]$ & $\delta$ (ring) \\
\hline \multirow[t]{2}{*}{$565 \mathrm{vw}$} & $570.7 \mathrm{vw}$ & & $577(5)[<1]$ & & & & $578(2)[<1]$ & $\gamma\left(\mathrm{N}_{3}\right)$ \\
\hline & & $516 \mathrm{~m}$ & $514(<1)[8]$ & & & $515 \mathrm{~m}$ & $513(<1)[8]$ & $\delta\left(\mathrm{N}_{3}\right)$ \\
\hline
\end{tabular}

${ }^{a}$ Calculated harmonic IR frequencies $\left(\mathrm{cm}^{-1} \text {, scaled by a factor of } 0.9679\right)^{1}$ and intensities (km mol ${ }^{-1}$, in parentheses) at the B3LYP/6-311++G(3df,3pd) level. ${ }^{b}$ Observed band positions of the most intense matrix sites in $\mathrm{N}_{2}$-matrix at $15 \mathrm{~K}$. The relative band intensities: vs $=$ very strong, $\mathrm{s}=\mathrm{strong}, \mathrm{m}=\mathrm{medium}$ strong, $\mathrm{w}=$ weak, vw $=$ very weak. ${ }^{c}$ Tentative assignment based on the calculated vibrational displacement vectors.

(1) Andersson, M. P.; Uvdal, P. New Scale Factors for Harmonic Vibrational Frequencies Using the B3LYP Density Functional Method with the Triple- $\xi$ Basis Set 6-311+G(d,p). J. Phys. Chem. A 2005, 109, 2937-2941. 
Table S2. Experimentally observed and calculated vibrational frequencies of pyridyl isocyanates 3 and 7.

\begin{tabular}{|c|c|c|c|c|}
\hline \multicolumn{3}{|c|}{ 3-pyridyl isocyanate (3) } & \multicolumn{2}{|c|}{ 4-pyridyl isocyanate (7) } \\
\hline Calculated $^{\mathrm{a}}$ & & Observed $^{\mathrm{b}}$ & Calculated $^{\mathrm{a}}$ & Observed $^{\mathrm{b}}$ \\
\hline anti & syn & $\mathrm{N}_{2}$-matrix & & $\mathrm{N}_{2}$-matrix \\
\hline $3096(7)$ & $3102(4)$ & & $3105(2)$ & \\
\hline 3080 (9) & 3088 (10) & $3076.5 \mathrm{vw}$ & 3091 (5) & \\
\hline 3067 (6) & $3063(7)$ & $3048.9 \mathrm{vw}$ & 3060 (9) & \\
\hline $3062(14)$ & 3047 (18) & $3034.9 \mathrm{vw}$ & 3057 (22) & $3049.8 \mathrm{vw}$ \\
\hline $2279(1728)$ & $2278(1754)$ & 2264.7 vs & 2277 (1709) & $2267.2 \mathrm{vs}$ \\
\hline $1575(32)$ & $1576(40)$ & $1591.5 \mathrm{~m}$ & $1576(248)$ & $1593.9 \mathrm{~s}$ \\
\hline $1551(2)$ & $1552(14)$ & $1575.4 \mathrm{vw}$ & $1546(42)$ & $1566.3 \mathrm{w}$ \\
\hline $1498(69)$ & $1500(57)$ & $1502.5 \mathrm{~m}$ & $1509(31)$ & $1520.3 \mathrm{w}$ \\
\hline $1441(<1)$ & $1436(1)$ & $1440.9 \mathrm{vw}$ & $1440(1)$ & \\
\hline $1399(30)$ & $1402(11)$ & $1412.0 \mathrm{w}$ & $1402(11)$ & $1417.3 \mathrm{w}$ \\
\hline $1316(1)$ & $1319(<1)$ & & $1314(<1)$ & \\
\hline $1235(8)$ & $1237(2)$ & $1236.2 \mathrm{vw}$ & $1229(5)$ & $1196.0 \mathrm{vw}$ \\
\hline $1182(4)$ & $1184(4)$ & $1210.4 \mathrm{vw}$ & $1204(8)$ & $1173.2 \mathrm{vw}$ \\
\hline $1125(51)$ & $1124(58)$ & $1160.2 \mathrm{w}$ & $1126(36)$ & $1132.6 \mathrm{vw}$ \\
\hline $1101(29)$ & 1098 (14) & $1121.1 \mathrm{w}$ & $1073(<1)$ & \\
\hline $1029(1)$ & $1029(2)$ & & $1052(<1)$ & \\
\hline $1005(13)$ & $1005(11)$ & $1022.6 \mathrm{~m}$ & $980(7)$ & $993.2 \mathrm{vw}$ \\
\hline $975(<1)$ & $978(<1)$ & & $979(<1)$ & \\
\hline $936(2)$ & $937(2)$ & $943.5 \mathrm{vw}$ & $953(<1)$ & \\
\hline $909(<1)$ & $903(<1)$ & & $844(2)$ & \\
\hline $795(27)$ & $801(29)$ & $805.8 \mathrm{~m}$ & $817(43)$ & $824,2 \mathrm{w}$ \\
\hline $769(8)$ & $767(4)$ & & $762(33)$ & $784.5 \mathrm{w}$ \\
\hline $702(29)$ & 702 (29) & $705.1 \mathrm{w}$ & $728(<1)$ & \\
\hline $639(33)$ & $640(24)$ & $654.1 \mathrm{~m}$ & $659(2)$ & \\
\hline $611(2)$ & $610(15)$ & $616.5 \mathrm{vw}$ & $632(37)$ & $648.3 \mathrm{w}$ \\
\hline 566 (19) & $566(20)$ & $572.6 \mathrm{~m}$ & $574(26)$ & $580.3 \mathrm{w}$ \\
\hline $488(<1)$ & $489(<1)$ & & 517 (12) & $520.7 \mathrm{vw}$ \\
\hline $466(<1)$ & $458(<1)$ & & $451(4)$ & \\
\hline $405(3)$ & $408(3)$ & & $382(<1)$ & \\
\hline $370(10)$ & 379 (18) & & 378 (10) & \\
\hline $231(<1)$ & $231(<1)$ & & $231(4)$ & \\
\hline 87 (4) & $90(<1)$ & & $89(2)$ & \\
\hline $58(<1)$ & $60(<1)$ & & $58(<1)$ & \\
\hline
\end{tabular}

${ }^{a}$ Calculated harmonic IR frequencies $\left(\mathrm{cm}^{-1} \text {, scaled by a factor of } 0.9679\right)^{1}$ and intensities $\left(\mathrm{km} \mathrm{mol}^{-1}\right.$, in parentheses) at the B3LYP/6-311++G(3df,3pd) level. ${ }^{b}$ Observed band positions of the most intense matrix sites in $\mathrm{N}_{2}$-matrix at $15 \mathrm{~K}$. The relative band intensities: $\mathrm{vs}=$ very strong, $\mathrm{s}=$ strong, $\mathrm{m}=$ medium strong, $\mathrm{w}=$ weak, $\mathrm{vW}=$ very weak. ${ }^{c}$ Tentative assignment based on the calculated vibrational displacement vectors. 
Table S3. Calculated vertical transitions for nicotinyl nitrene (2) at the TD-B3LYP/6-311++G(3df,3pd) level.

\begin{tabular}{|c|c|c|c|c|c|c|c|}
\hline \multicolumn{8}{|c|}{ nicotinyl nitrene (2) } \\
\hline \multicolumn{4}{|c|}{ singlet } & \multicolumn{4}{|c|}{ triplet } \\
\hline \multicolumn{2}{|c|}{ anti } & \multicolumn{2}{|c|}{ syn } & \multicolumn{2}{|c|}{ anti } & \multicolumn{2}{|c|}{ syn } \\
\hline $506 \mathrm{~nm}$ & $\mathrm{f}=0.0005$ & $504 \mathrm{~nm}$ & $\mathrm{f}=0.0005$ & $1115 \mathrm{~nm}$ & $f=0.0003$ & $1194 \mathrm{~nm}$ & $f=0.0002$ \\
\hline $310 \mathrm{~nm}$ & $\mathrm{f}=0.0450$ & $314 \mathrm{~nm}$ & $\mathrm{f}=0.0345$ & $547 \mathrm{~nm}$ & $\mathrm{f}=0.0300$ & $566 \mathrm{~nm}$ & $f=0.0164$ \\
\hline $292 \mathrm{~nm}$ & $f=0.0003$ & $295 \mathrm{~nm}$ & $\mathrm{f}=0.0004$ & $455 \mathrm{~nm}$ & $\mathrm{f}=0.0023$ & $474 \mathrm{~nm}$ & $\mathrm{f}=0.0008$ \\
\hline $275 \mathrm{~nm}$ & $f=0.0004$ & $275 \mathrm{~nm}$ & $f=0.0006$ & $415 \mathrm{~nm}$ & $f=0.0001$ & $408 \mathrm{~nm}$ & $f=0.0104$ \\
\hline $263 \mathrm{~nm}$ & $\mathrm{f}=0.0001$ & $260 \mathrm{~nm}$ & $f=0.0012$ & $351 \mathrm{~nm}$ & $f=0.0013$ & $338 \mathrm{~nm}$ & $\mathrm{f}=0.0025$ \\
\hline $261 \mathrm{~nm}$ & $f=0.0075$ & $256 \mathrm{~nm}$ & $f=0.0027$ & $324 \mathrm{~nm}$ & $f=0.0065$ & $327 \mathrm{~nm}$ & $\mathrm{f}=0.0013$ \\
\hline $256 \mathrm{~nm}$ & $f=0.0030$ & $250 \mathrm{~nm}$ & $f=0.0915$ & $320 \mathrm{~nm}$ & $f=0.0034$ & $326 \mathrm{~nm}$ & $f=0.0054$ \\
\hline $256 \mathrm{~nm}$ & $\mathrm{f}=0.0523$ & $234 \mathrm{~nm}$ & $f=0.0001$ & $313 \mathrm{~nm}$ & $f=0.0003$ & $315 \mathrm{~nm}$ & $\mathrm{f}=0.0023$ \\
\hline $234 \mathrm{~nm}$ & $f=0.0001$ & $228 \mathrm{~nm}$ & $f=0.1269$ & & & & \\
\hline $230 \mathrm{~nm}$ & $\mathrm{f}=0.1347$ & & & & & & \\
\hline
\end{tabular}

Table S4. Calculated vertical transitions for isonicotiny nitrene (6) and 4-pyridyl nitrene (8) at the TD-B3LYP/6-311++G(3df,3pd) level.

\begin{tabular}{cccccccc}
\hline & \multicolumn{2}{c}{ isonicotiny nitrene $(\mathbf{6})$} & \multicolumn{3}{c}{ 4-pyridyl nitrene $(\mathbf{8})$} \\
\multicolumn{2}{c}{ singlet } & \multicolumn{2}{c}{ triplet } & \multicolumn{2}{c}{ singlet } & \multicolumn{2}{c}{ triplet } \\
\hline $511 \mathrm{~nm}$ & $\mathrm{f}=0.0005$ & $1147 \mathrm{~nm}$ & $\mathrm{f}=0.0003$ & $839 \mathrm{~nm}$ & $\mathrm{f}=0.0072$ & $543 \mathrm{~nm}$ & $\mathrm{f}=0.0002$ \\
$319 \mathrm{~nm}$ & $\mathrm{f}=0.0336$ & $554 \mathrm{~nm}$ & $\mathrm{f}=0.0017$ & $360 \mathrm{~nm}$ & $\mathrm{f}=0.0001$ & $447 \mathrm{~nm}$ & $\mathrm{f}=0.0140$ \\
$308 \mathrm{~nm}$ & $\mathrm{f}=0.0018$ & $492 \mathrm{~nm}$ & $\mathrm{f}=0.0195$ & $291 \mathrm{~nm}$ & $\mathrm{f}=0.0109$ & $329 \mathrm{~nm}$ & $\mathrm{f}=0.0005$ \\
$282 \mathrm{~nm}$ & $\mathrm{f}=0.0007$ & $445 \mathrm{~nm}$ & $\mathrm{f}=0.0069$ & $272 \mathrm{~nm}$ & $\mathrm{f}=0.0050$ & $328 \mathrm{~nm}$ & $\mathrm{f}=0.0160$ \\
$272 \mathrm{~nm}$ & $\mathrm{f}=0.0001$ & $354 \mathrm{~nm}$ & $\mathrm{f}=0.0002$ & $269 \mathrm{~nm}$ & $\mathrm{f}=0.0238$ & $307 \mathrm{~nm}$ & $\mathrm{f}=0.0041$ \\
$256 \mathrm{~nm}$ & $\mathrm{f}=0.0471$ & $351 \mathrm{~nm}$ & $\mathrm{f}=0.0005$ & $247 \mathrm{~nm}$ & $\mathrm{f}=0.0003$ & $279 \mathrm{~nm}$ & $\mathrm{f}=0.0075$ \\
$234 \mathrm{~nm}$ & $\mathrm{f}=0.0215$ & $326 \mathrm{~nm}$ & $\mathrm{f}=0.0025$ & $240 \mathrm{~nm}$ & $\mathrm{f}=0.0001$ & $274 \mathrm{~nm}$ & $\mathrm{f}=0.0020$ \\
$209 \mathrm{~nm}$ & $\mathrm{f}=0.1323$ & $320 \mathrm{~nm}$ & $\mathrm{f}=0.0042$ & $236 \mathrm{~nm}$ & $\mathrm{f}=0.0023$ & $264 \mathrm{~nm}$ & $\mathrm{f}=0.0184$ \\
\hline
\end{tabular}


Calculated atomic coordinates (in Angstrom) and energies (in Hartree) for all optimized structures

anti-syn nicotinoyl azide (1)

B3LYP/6-311++G(3df,3pd)

$\begin{array}{lrrr}\mathrm{C} & 1.39357300 & 0.62976100 & 0.00000000 \\ \mathrm{C} & 0.00000000 & 0.53195500 & 0.00000000 \\ \mathrm{C} & -0.74552000 & 1.71112500 & 0.00000000 \\ \mathrm{C} & -0.07500000 & 2.92062500 & 0.00000000 \\ \mathrm{H} & 2.00107300 & -0.26525500 & 0.00000000 \\ \mathrm{H} & -1.82473900 & 1.65557400 & 0.00000000 \\ \mathrm{H} & -0.61189800 & 3.85845500 & 0.00000000 \\ \mathrm{C} & -0.72029600 & -0.76341500 & 0.00000000 \\ \mathrm{O} & -1.92249000 & -0.87171300 & 0.00000000 \\ \mathrm{~N} & 0.16443700 & -1.88346700 & 0.00000000 \\ \mathrm{~N} & -0.40019600 & -2.98777800 & 0.00000000 \\ \mathrm{~N} & -0.81915900 & -4.02561900 & 0.00000000 \\ \mathrm{C} & 1.31683600 & 2.90816100 & 0.00000000 \\ \mathrm{H} & 1.87270200 & 3.83862500 & 0.00000000 \\ \mathrm{~N} & 2.04423500 & 1.79072600 & 0.00000000\end{array}$

Zero-point correction $=$

0.101687

0.110377

0.111321

0.066746

$-525.296900$

$-525.288210$

$-525.287266$

$-525.331841$

Sum of electronic and thermal Free Energies=

\section{CBS-QB3}

$\begin{array}{lrrr}\mathrm{C} & 1.39704400 & 0.62633000 & 0.00000000 \\ \mathrm{C} & 0.00000000 & 0.53291300 & 0.00000000 \\ \mathrm{C} & -0.74420400 & 1.71660700 & 0.00000000 \\ \mathrm{C} & -0.06757200 & 2.92662500 & 0.00000000 \\ \mathrm{H} & 2.00096000 & -0.27428500 & 0.00000000 \\ \mathrm{H} & -1.82596500 & 1.66126800 & 0.00000000 \\ \mathrm{H} & -0.60180200 & 3.86892400 & 0.00000000 \\ \mathrm{C} & -0.72868900 & -0.76001800 & 0.00000000 \\ \mathrm{O} & -1.93292600 & -0.86374100 & 0.00000000 \\ \mathrm{~N} & 0.15584900 & -1.88347300 & 0.00000000 \\ \mathrm{~N} & -0.40637100 & -2.99181100 & 0.00000000 \\ \mathrm{~N} & -0.81993100 & -4.03730600 & 0.00000000 \\ \mathrm{C} & 1.32757100 & 2.90743900 & 0.00000000 \\ \mathrm{H} & 1.88887100 & 3.83769700 & 0.00000000 \\ \mathrm{~N} & 2.05565900 & 1.78644100 & 0.00000000\end{array}$

Temperature $=$

$\mathrm{E}(\mathrm{ZPE})=$

$\mathrm{E}(\mathrm{SCF})=$

$\mathrm{DE}(\mathrm{CBS})=$

$\mathrm{DE}(\mathrm{CCSD})=$

$\mathrm{DE}($ Empirical $)=$

CBS-QB3 $(0 \mathrm{~K})=$

CBS-QB3 Enthalpy=
1.000000

0.109360

$-1.934798$

$-0.018273$

0.059607

$-524.417127$

$-524.460784$

anti-anti nicotinoyl azide (1)

B3LYP/6-311++G(3df,3pd) 


$\begin{array}{rrrr}\mathrm{C} & 0.55496100 & -0.82872600 & -0.68340100 \\ \mathrm{C} & 0.24853800 & 0.40510600 & -0.10685500 \\ \mathrm{C} & 1.27309700 & 1.10333200 & 0.53294200 \\ \mathrm{C} & 2.52886600 & 0.52788200 & 0.60237300 \\ \mathrm{H} & -0.19343800 & -1.38530400 & -1.23491800 \\ \mathrm{H} & 1.06979600 & 2.07595700 & 0.95842700 \\ \mathrm{H} & 3.34652900 & 1.02936800 & 1.10029100 \\ \mathrm{C} & -1.09129500 & 1.05350500 & -0.22117800 \\ \mathrm{O} & -1.22579700 & 2.24014600 & -0.34791300 \\ \mathrm{~N} & -2.26606800 & 0.23839900 & -0.20728100 \\ \mathrm{~N} & -2.22917600 & -0.89536000 & 0.28391300 \\ \mathrm{~N} & -2.34771500 & -1.92372700 & 0.72113800 \\ \mathrm{C} & 2.72428300 & -0.71529200 & 0.00703400 \\ \mathrm{H} & 3.69566900 & -1.19446400 & 0.04263700 \\ \mathrm{~N} & 1.76540500 & -1.37953500 & -0.63614600\end{array}$

Zero-point correction=

0.101431

Thermal correction to Energy=

0.110126

0.111071

Thermal correction to Enthalpy=

0.066535

$-525.285084$

$-525.276389$

$-525.275444$

$-525.319980$

Sum of electronic and thermal Free Energies=

$\begin{array}{lrrc}\text { CBS-QB3 } & & & \\ \mathrm{C} & 0.54783700 & -0.85861900 & -0.64378100 \\ \mathrm{C} & 0.25605500 & 0.39865700 & -0.10286000 \\ \mathrm{C} & 1.29882600 & 1.11442100 & 0.49446400 \\ \mathrm{C} & 2.55431000 & 0.53122200 & 0.56576100 \\ \mathrm{H} & -0.21141100 & -1.43087600 & -1.16805700 \\ \mathrm{H} & 1.10624200 & 2.10619700 & 0.88496400 \\ \mathrm{H} & 3.38572900 & 1.04556000 & 1.03205700 \\ \mathrm{C} & -1.07959800 & 1.06338900 & -0.21470400 \\ \mathrm{O} & -1.20120900 & 2.25518100 & -0.31545900 \\ \mathrm{~N} & -2.26536200 & 0.25692300 & -0.23366100 \\ \mathrm{~N} & -2.25514100 & -0.87596700 & 0.26821600 \\ \mathrm{~N} & -2.40968000 & -1.90354400 & 0.70955600 \\ \mathrm{C} & 2.73012400 & -0.73792000 & 0.01324500 \\ \mathrm{H} & 3.69983100 & -1.22572000 & 0.05223500 \\ \mathrm{~N} & 1.75646100 & -1.42077100 & -0.59415100\end{array}$

Temperature $=$

$\mathrm{E}(\mathrm{ZPE})=$

$\mathrm{E}(\mathrm{SCF})=$

$\mathrm{DE}(\mathrm{CBS})=$

$\mathrm{DE}(\mathrm{CCSD})=$

$\mathrm{DE}($ Empirical $)=$

CBS-QB3 $(0 \mathrm{~K})=$

CBS-QB3 Enthalpy=

$$
\begin{gathered}
298.150000 \\
0.100298 \\
-522.277330 \\
-0.182130 \\
-0.075671 \\
-0.081237 \\
-524.414443 \\
-524.404727
\end{gathered}
$$

Pressure $=$

$\mathrm{E}($ Thermal $)=$

$\mathrm{DE}(\mathrm{MP} 2)=$

DE(MP34)=

$\mathrm{DE}($ Int $)=$

CBS-QB3 Energy=

CBS-QB3 Free Energy=

\author{
1.000000 \\ 0.109070 \\ $-1.942750$ \\ $-0.015623$ \\ 0.059999
}

$-524.405671$

$-524.449409$

syn-anti nicotinoyl azide (1)

B3LYP/6-311++G(3df,3pd)
C
$-1.25525300$
1.07627600
$-0.54261700$
C
$-0.23918200$
0.39051300
0.13141000
C
$-0.55124900$
$-0.83320500$
0.71899000 


$\begin{array}{lrrr}\mathrm{C} & -1.84636200 & -1.31480800 & 0.60673400 \\ \mathrm{H} & -1.04021000 & 2.03643700 & -0.99469800 \\ \mathrm{H} & 0.18769500 & -1.38922300 & 1.27939100 \\ \mathrm{H} & -2.13313000 & -2.25216800 & 1.06139300 \\ \mathrm{C} & 1.09825300 & 1.04309700 & 0.23620300 \\ \mathrm{O} & 1.23631000 & 2.22722300 & 0.37130000 \\ \mathrm{~N} & 2.27464500 & 0.22469300 & 0.19721000 \\ \mathrm{~N} & 2.22361100 & -0.90907700 & -0.28984700 \\ \mathrm{~N} & 2.32444900 & -1.94069000 & -0.72546400 \\ \mathrm{C} & -2.77629700 & -0.56207600 & -0.09991300 \\ \mathrm{H} & -3.79588400 & -0.91231800 & -0.20978300 \\ \mathrm{~N} & -2.49247800 & 0.61088800 & -0.66926200\end{array}$

Zero-point correction $=$

0.101382

Thermal correction to Energy=

Thermal correction to Enthalpy=

0.110094

0.111038

Thermal correction to Gibbs Free Energy=

Sum of electronic and zero-point Energies=

Sum of electronic and thermal Energies=

Sum of electronic and thermal Enthalpies=

Sum of electronic and thermal Free Energies=

0.066437

$-525.284650$

$-525.275938$

$-525.274994$

$-525.319595$

\section{CBS-QB3}

$\begin{array}{lrrr}\mathrm{C} & -1.28930900 & 1.08689600 & -0.49640100 \\ \mathrm{C} & -0.24810800 & 0.38205500 & 0.12466700 \\ \mathrm{C} & -0.53778100 & -0.87056300 & 0.67064300 \\ \mathrm{C} & -1.83268500 & -1.36238000 & 0.55983400 \\ \mathrm{H} & -1.09193900 & 2.07176500 & -0.90674600 \\ \mathrm{H} & 0.21598800 & -1.44356300 & 1.19716300 \\ \mathrm{H} & -2.10244600 & -2.32326700 & 0.98034600 \\ \mathrm{C} & 1.08286400 & 1.05499700 & 0.22752300 \\ \mathrm{O} & 1.20440000 & 2.24531600 & 0.33276100 \\ \mathrm{~N} & 2.27333400 & 0.24843400 & 0.22790500 \\ \mathrm{~N} & 2.25636100 & -0.88392600 & -0.27187500 \\ \mathrm{~N} & 2.39927500 & -1.91394700 & -0.71307500 \\ \mathrm{C} & -2.78507200 & -0.58850200 & -0.09892200 \\ \mathrm{H} & -3.80507700 & -0.94592600 & -0.20627900 \\ \mathrm{~N} & -2.52771100 & 0.61564700 & -0.62161800\end{array}$

Temperature $=$

$\mathrm{E}(\mathrm{ZPE})=$

$\mathrm{E}(\mathrm{SCF})=$

$\mathrm{DE}(\mathrm{CBS})=$

$\mathrm{DE}(\mathrm{CCSD})=$

$\mathrm{DE}($ Empirical $)=$

CBS-QB3 $(0 \mathrm{~K})=$

CBS-QB3 Enthalpy=

$$
\begin{gathered}
298.150000 \\
0.100235 \\
-522.276499 \\
-0.182176 \\
-0.075754 \\
-0.081226 \\
-524.414108 \\
-524.404373
\end{gathered}
$$

Pressure $=$

$\mathrm{E}($ Thermal $)=$

$\mathrm{DE}(\mathrm{MP} 2)=$

DE(MP34)=

$\mathrm{DE}(\operatorname{Int})=$

CBS-QB3 Energy=

CBS-QB3 Free Energy=
1.000000

0.109026

$-1.943232$

$-0.015470$

0.060012

$-524.405317$

$-524.449128$

syn-syn nicotinoyl azide (1)

B3LYP/6-311++G(3df,3pd)

$\begin{array}{lrrr}\mathrm{C} & -0.78974100 & 1.67703700 & 0.00000000 \\ \mathrm{C} & 0.00000000 & 0.52242500 & 0.00000000 \\ \mathrm{C} & 1.38592100 & 0.67063500 & 0.00000000 \\ \mathrm{C} & 1.91570500 & 1.95066900 & 0.00000000 \\ \mathrm{H} & -1.86794700 & 1.58248200 & 0.00000000 \\ \mathrm{H} & 2.02622800 & -0.19916300 & 0.00000000\end{array}$




$\begin{array}{lrrc}\mathrm{H} & 2.98423000 & 2.11247300 & 0.00000000 \\ \mathrm{C} & -0.67841400 & -0.79502100 & 0.00000000 \\ \mathrm{O} & -1.87461700 & -0.94951200 & 0.00000000 \\ \mathrm{~N} & 0.24975500 & -1.88384700 & 0.00000000 \\ \mathrm{~N} & -0.27522200 & -3.00729200 & 0.00000000 \\ \mathrm{~N} & -0.66214800 & -4.05758500 & 0.00000000 \\ \mathrm{C} & 1.04184000 & 3.03212700 & 0.00000000 \\ \mathrm{H} & 1.42498600 & 4.04604400 & 0.00000000 \\ \mathrm{~N} & -0.28701900 & 2.90687100 & 0.00000000\end{array}$

Zero-point correction=

0.101626

0.110331

0.111275

0.066629

$-525.296515$

$-525.287810$

$-525.286866$

$-525.331512$
Sum of electronic and thermal Energies=

Sum of electronic and thermal Enthalpies=

Sum of electronic and thermal Free Energies=

\section{CBS-QB3}

$\mathrm{C}$
$\mathrm{C}$
$\mathrm{C}$
$\mathrm{C}$
$\mathrm{H}$
$\mathrm{H}$
$\mathrm{H}$
$\mathrm{C}$
$\mathrm{O}$
$\mathrm{N}$
$\mathrm{N}$
$\mathrm{N}$
$\mathrm{C}$
$\mathrm{H}$
$\mathrm{N}$

Temperature $=$

$\mathrm{E}(\mathrm{ZPE})=$

$\mathrm{E}(\mathrm{SCF})=$

$\mathrm{DE}(\mathrm{CBS})=$

$\mathrm{DE}(\mathrm{CCSD})=$

$\mathrm{DE}($ Empirical $)=$

CBS-QB3 $(0 \mathrm{~K})=$

CBS-QB3 Enthalpy=

-0.78810700
0.00000000
1.38979600
1.92300700
-1.86881000
2.02661600
2.99414700
-0.68548800
-1.88366100
0.24340700
-0.27778900
-0.65867100
1.04836800
1.43425100
-0.28442800

1.68234200

0.52327100

0.66676200

1.94886500

1.58787000

$-0.20866400$

2.10984000

$-0.79266400$

$-0.94365200$

$-1.88464900$

$-3.01240800$

$-4.07042900$

3.03412600

4.04970000

2.91523600
0.00000000

0.00000000

0.00000000

0.00000000

0.00000000

0.00000000

0.00000000

0.00000000

0.00000000

0.00000000

0.00000000

0.00000000

0.00000000

0.00000000

0.00000000

anti nicotinoyl nitrene-singlet (2)

\section{B3LYP/6-311++G(3df,3pd)}

$\begin{array}{lrcc}\mathrm{C} & 1.30027900 & -0.28280900 & 0.00000000 \\ \mathrm{C} & 0.00000000 & 0.22941300 & 0.00000000 \\ \mathrm{C} & -1.07563300 & -0.65613300 & 0.00000000 \\ \mathrm{C} & -0.80402600 & -2.01387600 & 0.00000000 \\ \mathrm{H} & 2.15121900 & 0.38884900 & 0.00000000 \\ \mathrm{H} & -2.08877800 & -0.27781400 & 0.00000000 \\ \mathrm{H} & -1.60058200 & -2.74380100 & 0.00000000 \\ \mathrm{C} & -0.24044000 & 1.65505300 & 0.00000000 \\ \mathrm{O} & -1.35275600 & 2.34859800 & 0.00000000\end{array}$

\author{
1.000000 \\ 0.109325 \\ $-1.934891$ \\ $-0.018311$ \\ 0.059603 \\ $-524.416740$ \\ $-524.460458$
}

CBS-QB3 Energy=

CBS-QB3 Free Energy= 


$\begin{array}{lrrr}\mathrm{C} & 0.52523700 & -2.42482500 & 0.00000000 \\ \mathrm{H} & 0.77040600 & -3.48031800 & 0.00000000 \\ \mathrm{~N} & 1.56246600 & -1.58323700 & 0.00000000 \\ \mathrm{~N} & 0.34571700 & 2.76657400 & 0.00000000\end{array}$

Zero-point correction $=$

0.089966

Thermal correction to Energy=

0.097077

0.098021

Thermal correction to Enthalpy=

0.057810

Thermal correction to Gibbs Free Energy=

Sum of electronic and zero-point Energies=

Sum of electronic and thermal Energies=

Sum of electronic and thermal Enthalpies=

Sum of electronic and thermal Free Energies=

$-415.698364$

$-415.691253$

$-415.690309$

$-415.730520$

\section{CBS-QB3}

$\begin{array}{lrrc}\mathrm{C} & 1.30597900 & -0.27252700 & 0.00000000 \\ \mathrm{C} & 0.00000000 & 0.23219700 & 0.00000000 \\ \mathrm{C} & -1.07201900 & -0.66232500 & 0.00000000 \\ \mathrm{C} & -0.79013500 & -2.02108700 & 0.00000000 \\ \mathrm{H} & 2.15439200 & 0.40636800 & 0.00000000 \\ \mathrm{H} & -2.08963300 & -0.28931400 & 0.00000000 \\ \mathrm{H} & -1.58276700 & -2.75887600 & 0.00000000 \\ \mathrm{C} & -0.25690300 & 1.65851900 & 0.00000000 \\ \mathrm{O} & -1.38229000 & 2.33081700 & 0.00000000 \\ \mathrm{C} & 0.54560400 & -2.42239500 & 0.00000000 \\ \mathrm{H} & 0.79986300 & -3.47824900 & 0.00000000 \\ \mathrm{~N} & 1.58106500 & -1.57367700 & 0.00000000 \\ \mathrm{~N} & 0.33055100 & 2.77356900 & 0.00000000\end{array}$

Temperature $=$

298.150000 Pressure $=$

1.000000

$\mathrm{E}(\mathrm{ZPE})=$

0.088897

$\mathrm{E}($ Thermal $)=$

0.096096

$-413.291074 \quad$ DE $(M P 2)=$

$-1.538187$

$-0.144817 \quad$ DE(MP34) $=$

$-0.032397$

$\mathrm{DE}(\mathrm{CBS})=$

$-0.065378$

$\mathrm{DE}(\mathrm{Int})=$

0.048146

$\mathrm{DE}($ Empirical $)=$ $-0.065586$

CBS-QB3 $(0 \mathrm{~K})=$

$-415.000397$

CBS-QB3 Energy=

$-414.993198$

CBS-QB3 Enthalpy=

$-414.992253$

CBS-QB3 Free Energy=

$-415.032687$

syn nicotinoyl nitrene-singlet (2)

\section{B3LYP/6-311++G(3df,3pd)}

$\begin{array}{lrrr}\mathrm{C} & -0.98195700 & -0.75805300 & 0.00000000 \\ \mathrm{C} & 0.00000000 & 0.23427200 & 0.00000000 \\ \mathrm{C} & 1.34318400 & -0.14283600 & 0.00000000 \\ \mathrm{C} & 1.64140200 & -1.49273000 & 0.00000000 \\ \mathrm{H} & -2.02963800 & -0.48229500 & 0.00000000 \\ \mathrm{H} & 2.12329200 & 0.60644200 & 0.00000000 \\ \mathrm{H} & 2.66458200 & -1.83972200 & 0.00000000 \\ \mathrm{C} & -0.38991700 & 1.62626300 & 0.00000000 \\ \mathrm{O} & -1.56164900 & 2.21205600 & 0.00000000 \\ \mathrm{C} & 0.58945800 & -2.40680200 & 0.000000000 \\ \mathrm{H} & 0.79276900 & -3.47128800 & 0.00000000 \\ \mathrm{~N} & -0.69700800 & -2.05667900 & 0.00000000 \\ \mathrm{~N} & 0.08689100 & 2.78949700 & 0.00000000\end{array}$

Zero-point correction=

0.090000

Thermal correction to Energy=

0.097103 
Thermal correction to Enthalpy=

0.098047

Thermal correction to Gibbs Free Energy=

Sum of electronic and zero-point Energies=

Sum of electronic and thermal Energies=

Sum of electronic and thermal Enthalpies=

Sum of electronic and thermal Free Energies=

0.057857

$-415.698024$

$-415.690921$

$-415.689977$

$-415.730167$

\section{CBS-QB3}

$\begin{array}{lrrc}\mathrm{C} & -0.97455500 & -0.76584100 & 0.00000000 \\ \mathrm{C} & 0.00000000 & 0.23746900 & 0.00000000 \\ \mathrm{C} & 1.34941700 & -0.12827200 & 0.00000000 \\ \mathrm{C} & 1.65941700 & -1.47906500 & 0.00000000 \\ \mathrm{H} & -2.02671600 & -0.49783500 & 0.00000000 \\ \mathrm{H} & 2.12489300 & 0.62931800 & 0.00000000 \\ \mathrm{H} & 2.68755100 & -1.81900500 & 0.00000000 \\ \mathrm{C} & -0.40820200 & 1.62785600 & 0.00000000 \\ \mathrm{O} & -1.59220200 & 2.18882000 & 0.00000000 \\ \mathrm{C} & 0.61190100 & -2.40304000 & 0.00000000 \\ \mathrm{H} & 0.82373200 & -3.46835600 & 0.00000000 \\ \mathrm{~N} & -0.68147300 & -2.06559800 & 0.00000000 \\ \mathrm{~N} & 0.06722900 & 2.79569500 & 0.00000000\end{array}$

Temperature $=$

$\mathrm{E}(\mathrm{ZPE})=$

$\mathrm{E}(\mathrm{SCF})=$

$\mathrm{DE}(\mathrm{CBS})=$

$\mathrm{DE}(\mathrm{CCSD})=$

$\mathrm{DE}($ Empirical $)=$

CBS-QB3 $(0 \mathrm{~K})=$

298.150000

Pressure $=$

1.000000

0.088909

$\mathrm{E}($ Thermal $)=$

0.096106

$-413.290543$

$\mathrm{DE}(\mathrm{MP} 2)=$

$-1.538742$

$-0.144805$

$\mathrm{DE}(\mathrm{MP} 34)=$

$-0.032190$

$-0.065334$

$\mathrm{DE}(\operatorname{Int})=$

0.048143

$-0.065579$

CBS-QB3 Enthalpy=

$-415.000141$

CBS-QB3 Energy=

$-414.992944$

$-414.992000$

CBS-QB3 Free Energy=

$-415.032429$

anti nicotinoyl nitrene-triplet (2)

B3LYP/6-311++G(3df,3pd)

$\begin{array}{lrrr}\mathrm{C} & 0.44553600 & -1.19080700 & 0.00000700 \\ \mathrm{C} & -0.27754200 & 0.00508000 & 0.00000000 \\ \mathrm{C} & 0.42797800 & 1.20878500 & -0.00000400 \\ \mathrm{C} & 1.80964700 & 1.16343400 & -0.00000100 \\ \mathrm{H} & -0.07245200 & -2.14275800 & 0.00000900 \\ \mathrm{H} & -0.11341200 & 2.14455000 & -0.00000900 \\ \mathrm{H} & 2.40018300 & 2.06856200 & -0.00000300 \\ \mathrm{C} & -1.75949000 & 0.01554600 & -0.00000400 \\ \mathrm{O} & -2.44289700 & 1.03954700 & -0.00001100 \\ \mathrm{C} & 2.43483100 & -0.08238300 & 0.00000500 \\ \mathrm{H} & 3.51652600 & -0.15060800 & 0.00000900 \\ \mathrm{~N} & 1.77484100 & -1.24040200 & 0.00000900 \\ \mathrm{~N} & -2.44247600 & -1.18160600 & 0.00000000\end{array}$

Zero-point correction=

0.089216

Thermal correction to Energy=

0.096275

Thermal correction to Enthalpy=

0.097219

Thermal correction to Gibbs Free Energy=

Sum of electronic and zero-point Energies=

Sum of electronic and thermal Energies=

0.055984

$-415.705327$

$-415.698268$

$-415.697324$

$-415.738559$ 


$\begin{array}{lrrc}\text { CBS-QB3 } & & & \\ \mathrm{C} & -0.44970300 & -1.19398200 & 0.00000900 \\ \mathrm{C} & 0.27857500 & 0.00199200 & 0.00002000 \\ \mathrm{C} & -0.42462800 & 1.21036800 & 0.00001700 \\ \mathrm{C} & -1.80982200 & 1.16842400 & 0.00000100 \\ \mathrm{H} & 0.06742700 & -2.14933200 & 0.00000700 \\ \mathrm{H} & 0.12323600 & 2.14513800 & 0.00002700 \\ \mathrm{H} & -2.39984600 & 2.07681000 & -0.00000200 \\ \mathrm{C} & 1.76332500 & 0.01368900 & 0.00003500 \\ \mathrm{O} & 2.44417400 & 1.04265400 & -0.00001800 \\ \mathrm{C} & -2.43820700 & -0.07910200 & -0.00001300 \\ \mathrm{H} & -3.52249100 & -0.14578200 & -0.00002500 \\ \mathrm{~N} & -1.78209000 & -1.24330500 & -0.00000900 \\ \mathrm{~N} & 2.44795200 & -1.18475200 & -0.00003100\end{array}$

Temperature $=$

$\mathrm{E}(\mathrm{ZPE})=$

$\mathrm{E}(\mathrm{SCF})=$

$\mathrm{DE}(\mathrm{CBS})=$

$\mathrm{DE}(\mathrm{CCSD})=$

$\mathrm{DE}($ Empirical $)=$

CBS-QB3 $(0 \mathrm{~K})=$

CBS-QB3 Enthalpy=

298.150000
0.088283
-413.378991
-0.136901
-0.079889
-0.068542
-414.993304
-414.985275

Pressure $=$

$\mathrm{E}($ Thermal $)=$

$\mathrm{DE}(\mathrm{MP} 2)=$

$\mathrm{DE}(\mathrm{MP} 34)=$

$\mathrm{DE}(\mathrm{Int})=$

CBS-QB3 Energy=

CBS-QB3 Free Energy=
1.000000 0.095368

$-1.387868$

$-0.071885$

0.042489

$-414.986219$

$-415.026463$

\section{syn nicotinoyl nitrene-triplet (2)}

\section{B3LYP/6-311++G(3df,3pd)}

$\mathrm{C}$
$\mathrm{C}$
$\mathrm{C}$
$\mathrm{C}$
$\mathrm{H}$
$\mathrm{H}$
$\mathrm{H}$
$\mathrm{C}$
$\mathrm{O}$
$\mathrm{C}$
$\mathrm{H}$
$\mathrm{N}$
$\mathrm{N}$

0.43290100
-0.27796200
0.44270600
1.82647600
-0.10694700
-0.07116600
2.42571100
-1.75947200
-2.44356000
2.43527100
3.51588000
1.75945900
-2.44724600

$\begin{array}{cc}-1.18460900 & -0.00000500 \\ 0.02036100 & -0.00000800 \\ 1.21506500 & 0.00001900 \\ 1.15436900 & 0.00003800 \\ -2.12304000 & -0.00003800 \\ 2.16663000 & 0.00002600 \\ 2.05357300 & 0.00006500 \\ -0.00086700 & -0.00003600 \\ -1.02416900 & -0.00002400 \\ -0.09577900 & 0.00001800 \\ -0.17833100 & 0.00004400 \\ -1.24790500 & 0.00000200 \\ 1.19408800 & -0.00001000\end{array}$

Zero-point correction $=$

0.089174

0.096252

0.097196

Thermal correction to Enthalpy=

Thermal correction to Gibbs Free Energy=

Sum of electronic and zero-point Energies=

Sum of electronic and thermal Energies=

Sum of electronic and thermal Enthalpies=

Sum of electronic and thermal Free Energies=

0.055831

$-415.704593$

$-415.697515$

$-415.696571$

$-415.737936$

\section{CBS-QB3}

$\begin{array}{lrrc}\mathrm{C} & 0.43204300 & -1.18396700 & -0.00003900 \\ \mathrm{C} & -0.27905000 & 0.02393300 & -0.00001100 \\ \mathrm{C} & 0.44422500 & 1.22077700 & 0.00001800 \\ \mathrm{C} & 1.83112100 & 1.15673800 & 0.00003700 \\ \mathrm{H} & -0.11244200 & -2.12250600 & -0.00003700 \\ \mathrm{H} & -0.07005100 & 2.17492000 & 0.00002200\end{array}$




$\begin{array}{lrrr}\mathrm{H} & 2.43419000 & 2.05634200 & 0.00004700 \\ \mathrm{C} & -1.76346200 & -0.00016400 & -0.00002800 \\ \mathrm{O} & -2.44345600 & -1.02951800 & -0.00002900 \\ \mathrm{C} & 2.43816800 & -0.09809600 & 0.00005500 \\ \mathrm{H} & 3.52105900 & -0.18329300 & 0.00003700 \\ \mathrm{~N} & 1.76160700 & -1.25344400 & 0.00000200 \\ \mathrm{~N} & -2.45351800 & 1.19563700 & -0.00000500\end{array}$

Temperature $=$

$\mathrm{E}(\mathrm{ZPE})=$

$\mathrm{E}(\mathrm{SCF})=$

$\mathrm{DE}(\mathrm{CBS})=$

$\mathrm{DE}(\mathrm{CCSD})=$

$\mathrm{DE}($ Empirical $)=$

CBS-QB3 $(0 \mathrm{~K})=$

CBS-QB3 Enthalpy=

$$
\begin{gathered}
298.150000 \\
0.088243 \\
-413.378688 \\
-0.136839 \\
-0.080074 \\
-0.068644 \\
-414.992659 \\
-414.984615
\end{gathered}
$$

Pressure $=$

$\mathrm{E}($ Thermal $)=$

DE $($ MP2 $)=$

$\mathrm{DE}(\mathrm{MP} 34)=$

$\mathrm{DE}($ Int $)=$

CBS-QB3 Energy=

CBS-QB3 Free Energy=
1.000000

0.095343

$-1.386902$

$-0.072215$

0.042460

$-414.985559$

$-415.025883$

anti 3-pyridyl isocyanate (3)

\section{B3LYP/6-311++G(3df,3pd)}

$\begin{array}{lrrr}\mathrm{C} & 0.87166900 & -1.25688200 & 0.00002000 \\ \mathrm{C} & -0.08237900 & -0.23689500 & 0.00001800 \\ \mathrm{C} & 0.36036400 & 1.08448800 & 0.00001400 \\ \mathrm{C} & 1.72533500 & 1.32162100 & 0.00000400 \\ \mathrm{H} & 0.54447200 & -2.28972500 & 0.00001200 \\ \mathrm{H} & -0.35008100 & 1.90026200 & 0.00001800 \\ \mathrm{H} & 2.10949300 & 2.33203700 & 0.00000100 \\ \mathrm{C} & 2.59651800 & 0.23823800 & -0.00000600 \\ \mathrm{H} & 3.66865400 & 0.39267600 & -0.00001200 \\ \mathrm{~N} & 2.17983900 & -1.02795500 & 0.00000200 \\ \mathrm{~N} & -1.42976400 & -0.58482100 & 0.00002900 \\ \mathrm{C} & -2.51655700 & -0.07183200 & -0.00003500 \\ \mathrm{O} & -3.61909600 & 0.31022000 & -0.00004100\end{array}$

Zero-point correction=

0.091903

0.098868

Thermal correction to Energy=

0.099813

Thermal correction to Enthalpy=

Thermal correction to Gibbs Free Energy=

Sum of electronic and zero-point Energies=

Sum of electronic and thermal Energies=

Sum of electronic and thermal Enthalpies=

Sum of electronic and thermal Free Energies=

0.059515

$-415.822056$

$-415.815091$

$-415.814147$

$-415.854444$

\section{CBS-QB3}

$\begin{array}{lrrr}\mathrm{C} & 0.87307700 & -1.25820700 & 0.00005000 \\ \mathrm{C} & -0.08279600 & -0.23514700 & 0.00004300 \\ \mathrm{C} & 0.36161100 & 1.08937300 & 0.00003400 \\ \mathrm{C} & 1.73027900 & 1.32471000 & 0.00000800 \\ \mathrm{H} & 0.54279300 & -2.29249900 & 0.00000700 \\ \mathrm{H} & -0.34938700 & 1.90790400 & 0.00004000 \\ \mathrm{H} & 2.11702400 & 2.33684100 & -0.00000400 \\ \mathrm{C} & 2.60097900 & 0.23661800 & -0.00001800 \\ \mathrm{H} & 3.67587000 & 0.38930100 & -0.00004600 \\ \mathrm{~N} & 2.18480100 & -1.03307000 & -0.00000800 \\ \mathrm{~N} & -1.43277800 & -0.58446500 & 0.00004500 \\ \mathrm{C} & -2.52224600 & -0.07177800 & -0.00005100 \\ \mathrm{O} & -3.62698600 & 0.30847300 & -0.00008000\end{array}$




$\begin{array}{lclc}\text { Temperature }= & 298.150000 & \text { Pressure= } & 1.000000 \\ \mathrm{E}(\mathrm{ZPE})= & 0.090869 & \mathrm{E}(\text { Thermal })= & 0.097906 \\ \mathrm{E}(\mathrm{SCF})= & -413.427925 & \mathrm{DE}(\mathrm{MP})= & -1.532845 \\ \mathrm{DE}(\mathrm{CBS})= & -0.145533 & \mathrm{DE}(\mathrm{MP} 34)= & -0.021569 \\ \mathrm{DE}(\mathrm{CCSD})= & -0.059100 & \mathrm{DE}(\mathrm{Int})= & 0.048116 \\ \text { DE(Empirical })= & -0.066257 & & \\ \text { CBS-QB3 }(0 \mathrm{~K})= & -415.114243 & \text { CBS-QB3 Energy }= & -415.107206 \\ \text { CBS-QB3 Enthalpy }= & -415.106261 & \text { CBS-QB3 Free Energy }= & -415.146716\end{array}$

syn 3-pyridyl isocyanate (3)

\section{B3LYP/6-311++G(3df,3pd)}

$\begin{array}{lrrc}\mathrm{C} & 0.35866100 & -1.05387800 & -0.00000100 \\ \mathrm{C} & -0.07655800 & 0.27612800 & 0.00000200 \\ \mathrm{C} & 0.88199600 & 1.28438700 & 0.00000300 \\ \mathrm{C} & 2.22041700 & 0.92416600 & 0.00000100 \\ \mathrm{H} & -0.36834100 & -1.85936200 & -0.00000300 \\ \mathrm{H} & 0.57173700 & 2.31963300 & 0.00000600 \\ \mathrm{H} & 2.99486900 & 1.67822300 & 0.00000200 \\ \mathrm{C} & 2.55339200 & -0.42477900 & -0.00000400 \\ \mathrm{H} & 3.58990500 & -0.73906200 & -0.00000500 \\ \mathrm{~N} & 1.63967600 & -1.39659900 & -0.00000400 \\ \mathrm{~N} & -1.42683300 & 0.61103200 & 0.00000300 \\ \mathrm{C} & -2.50776600 & 0.08427000 & 0.00000200 \\ \mathrm{O} & -3.60736500 & -0.30527800 & 0.00000000\end{array}$

Zero-point correction $=$

0.091928

0.098875

0.099819

Thermal correction to Enthalpy=

0.059601

Thermal correction to Gibbs Free Energy=

$-415.822560$

$-415.815612$

$-415.814668$

$-415.854887$

Sum of electronic and thermal Enthalpies=

Sum of electronic and thermal Free Energies=

\section{CBS-QB3}

$\begin{array}{lrrr}\mathrm{C} & 0.35895900 & -1.05561000 & 0.00000000 \\ \mathrm{C} & -0.07747800 & 0.27739700 & 0.00000500 \\ \mathrm{C} & 0.88314800 & 1.28850800 & 0.00000500 \\ \mathrm{C} & 2.22431200 & 0.92642300 & 0.00000000 \\ \mathrm{H} & -0.36911700 & -1.86345600 & 0.00000200 \\ \mathrm{H} & 0.57066100 & 2.32540300 & 0.00000800 \\ \mathrm{H} & 3.00149200 & 1.68123200 & -0.00000200 \\ \mathrm{C} & 2.55612600 & -0.42634300 & -0.00000400 \\ \mathrm{H} & 3.59464800 & -0.74248900 & -0.00000900 \\ \mathrm{~N} & 1.64232300 & -1.40227500 & -0.00000400 \\ \mathrm{~N} & -1.43009200 & 0.61705800 & 0.00001100 \\ \mathrm{C} & -2.51105000 & 0.08410800 & -0.00000100 \\ \mathrm{O} & -3.61092500 & -0.30888200 & -0.00001000\end{array}$

Temperature $=$

$\mathrm{E}(\mathrm{ZPE})=$

$\mathrm{E}(\mathrm{SCF})=$

$\mathrm{DE}(\mathrm{CBS})=$

$\mathrm{DE}(\mathrm{CCSD})=$

$\mathrm{DE}($ Empirical $)=$

$$
\begin{array}{cl}
298.150000 & \text { Pressure }= \\
0.090898 & \mathrm{E}(\text { Thermal })= \\
-413.428169 & \text { DE(MP2)= } \\
-0.145554 & \text { DE }(\text { MP34 })= \\
-0.059113 & \text { DE }(\text { Int })= \\
-0.066254 &
\end{array}
$$

1.000000
0.097911
-1.532977
-0.021517
0.048126 


$\begin{array}{lllc}\text { CBS-QB3 }(0 \mathrm{~K})= & -415.114560 & \text { CBS-QB3 Energy }= & -415.107547 \\ \text { CBS-QB3 Enthalpy }= & -415.106603 & \text { CBS-QB3 Free Energy }= & -415.146953\end{array}$

$\begin{array}{lrcc}\text { anti isonicotinoyl azide (5) } & & \\ \text { B3LYP/6-311++G(3df,3pd) } & & \\ \mathrm{C} & 0.55425800 & -0.80672500 & -0.73113700 \\ \mathrm{C} & 0.23565100 & 0.40524700 & -0.12516200 \\ \mathrm{C} & 1.23825700 & 1.08440100 & 0.56234800 \\ \mathrm{C} & 2.49934500 & 0.50945300 & 0.63525500 \\ \mathrm{H} & -0.17095100 & -1.36366500 & -1.30719700 \\ \mathrm{H} & 1.03267400 & 2.03923200 & 1.02374200 \\ \mathrm{H} & 3.29515200 & 1.00702100 & 1.17650400 \\ \mathrm{C} & -1.11403500 & 1.04715400 & -0.23647700 \\ \mathrm{O} & -1.25425800 & 2.22710800 & -0.40025500 \\ \mathrm{~N} & -2.28065700 & 0.22683300 & -0.16809100 \\ \mathrm{~N} & -2.21126500 & -0.91404500 & 0.30456600 \\ \mathrm{~N} & -2.30299800 & -1.94967700 & 0.72995800 \\ \mathrm{~N} & 2.81299100 & -0.65170100 & 0.05841900 \\ \mathrm{C} & 1.85563400 & -1.28343300 & -0.61694700 \\ \mathrm{H} & 2.13603500 & -2.21589500 & -1.09224600\end{array}$

Zero-point correction=

0.101345

0.110052

0.110997

Thermal correction to Enthalpy=

0.066345

$-525.283945$

$-525.275237$

$-525.274293$

$-525.318945$

Sum of electronic and thermal Free Energies=

$\begin{array}{lrrc}\text { CBS-QB3 } & & & \\ \mathrm{C} & 0.53945800 & -0.85012600 & -0.67333500 \\ \mathrm{C} & 0.24533800 & 0.39584900 & -0.11704600 \\ \mathrm{C} & 1.27538100 & 1.09860900 & 0.51111400 \\ \mathrm{C} & 2.53411700 & 0.51181600 & 0.58342200 \\ \mathrm{H} & -0.20237300 & -1.42892100 & -1.20906300 \\ \mathrm{H} & 1.08841300 & 2.08061100 & 0.92624600 \\ \mathrm{H} & 3.35042600 & 1.02756900 & 1.08026100 \\ \mathrm{C} & -1.09700100 & 1.06069800 & -0.22678900 \\ \mathrm{O} & -1.21746700 & 2.24818100 & -0.35886300 \\ \mathrm{~N} & -2.28027900 & 0.25428400 & -0.20120100 \\ \mathrm{~N} & -2.24883600 & -0.88593600 & 0.28354000 \\ \mathrm{~N} & -2.38593900 & -1.92088200 & 0.71275600 \\ \mathrm{~N} & 2.82501900 & -0.68380100 & 0.05966600 \\ \mathrm{C} & 1.84125700 & -1.33590300 & -0.56268000 \\ \mathrm{H} & 2.10220700 & -2.29602400 & -0.99798200\end{array}$

Temperature $=$

$\mathrm{E}(\mathrm{ZPE})=$

$\mathrm{E}(\mathrm{SCF})=$

$\mathrm{DE}(\mathrm{CBS})=$

$\mathrm{DE}(\mathrm{CCSD})=$

$\mathrm{DE}($ Empirical $)=$

CBS-QB3 $(0 \mathrm{~K})=$

CBS-QB3 Enthalpy=
298.150000 Pressure $=$

$0.100193 \quad \mathrm{E}($ Thermal $)=$

$-522.274765$

$-0.182531$

$-0.075627$

$-0.081170$

$-524.413729$

$-524.404003$
$\mathrm{DE}(\mathrm{MP} 2)=$

DE(MP34) $=$

$\mathrm{DE}($ Int $)=$

CBS-QB3 Energy=

CBS-QB3 Free Energy=
1.000000

0.108976

$-1.945375$

$-0.014585$

0.060132

$-524.404947$

$-524.448778$ 
syn isonicotinoyl azide (5)

B3LYP/6-311++G(3df,3pd)

$\begin{array}{lrrr}\mathrm{C} & -1.42457700 & 1.18764600 & 0.00009500 \\ \mathrm{C} & -0.50573100 & 0.14075000 & -0.00001300 \\ \mathrm{C} & -0.98810600 & -1.16530100 & -0.00010000 \\ \mathrm{C} & -2.36401400 & -1.36198800 & -0.00007600 \\ \mathrm{H} & -1.08009100 & 2.21120700 & 0.00015400 \\ \mathrm{H} & -0.31255100 & -2.00704800 & -0.00018300 \\ \mathrm{H} & -2.77019500 & -2.36649000 & -0.00016600 \\ \mathrm{C} & 0.94674900 & 0.47040000 & -0.00006100 \\ \mathrm{O} & 1.38607100 & 1.59311900 & -0.00022400 \\ \mathrm{~N} & 1.76901900 & -0.69424200 & 0.00011400 \\ \mathrm{~N} & 2.98877700 & -0.46492400 & 0.00007700 \\ \mathrm{~N} & 4.10233400 & -0.35600100 & 0.00006600 \\ \mathrm{~N} & -3.24964500 & -0.36669500 & 0.00003000 \\ \mathrm{C} & -2.77797700 & 0.88133200 & 0.00011600 \\ \mathrm{H} & -3.51719300 & 1.67337700 & 0.00021800\end{array}$

Zero-point correction $=$

0.101647

Thermal correction to Energy=

0.110318

Thermal correction to Enthalpy=

0.111262

Thermal correction to Gibbs Free Energy=

Sum of electronic and zero-point Energies=

0.066681

$-525.295387$

$-525.286716$

$-525.285772$

Sum of electronic and thermal Enthalpies=

$-525.330353$

\section{CBS-QB3}

$\begin{array}{lrrr}\mathrm{C} & -1.42828000 & 1.18965200 & -0.00001500 \\ \mathrm{C} & -0.50599300 & 0.14145000 & 0.00013700 \\ \mathrm{C} & -0.98654400 & -1.16879200 & 0.00013900 \\ \mathrm{C} & -2.36539200 & -1.36620600 & -0.00002000 \\ \mathrm{H} & -1.08139800 & 2.21493500 & -0.00003300 \\ \mathrm{H} & -0.30587800 & -2.00962600 & 0.00026900 \\ \mathrm{H} & -2.77229900 & -2.37317200 & -0.00003200 \\ \mathrm{C} & 0.94671100 & 0.47759800 & 0.00030400 \\ \mathrm{O} & 1.38396100 & 1.60281200 & 0.00005500 \\ \mathrm{~N} & 1.77111300 & -0.68909800 & 0.00008900 \\ \mathrm{~N} & 2.99424500 & -0.46547300 & -0.00009000 \\ \mathrm{~N} & 4.11387300 & -0.36577900 & -0.00025600 \\ \mathrm{~N} & -3.25710900 & -0.37165200 & -0.00014900 \\ \mathrm{C} & -2.78383700 & 0.87932400 & -0.00014100 \\ \mathrm{H} & -3.52695300 & 1.67123200 & -0.00023500\end{array}$

Temperature $=$

$\mathrm{E}(\mathrm{ZPE})=$

$\mathrm{E}(\mathrm{SCF})=$

$\mathrm{DE}(\mathrm{CBS})=$

$\mathrm{DE}(\mathrm{CCSD})=$

$\mathrm{DE}($ Empirical $)=$

CBS-QB3 $(0 \mathrm{~K})=$

CBS-QB3 Enthalpy=

$\begin{array}{cl}298.150000 & \text { Pressure }= \\ 0.100541 & \mathrm{E}(\text { Thermal })= \\ -522.291848 & \text { DE(MP2)= } \\ -0.182251 & \text { DE }(\mathrm{MP} 34)= \\ -0.074495 & \text { DE(Int })= \\ -0.081337 & \\ -524.424836 & \text { CBS-QB3 Energy }= \\ -524.415151 & \text { CBS-QB3 Free Energy }=\end{array}$

1.000000

0.109282

$-1.938469$

$-0.016796$

0.059818

$-524.416096$

$-524.459784$

isonicotinoyl nitrene-singlet (6)

B3LYP/6-311++G(3df,3pd) 


$\begin{array}{lrrr}\mathrm{C} & 0.42203300 & -1.17170100 & -0.00032000 \\ \mathrm{C} & -0.22898200 & 0.05761300 & 0.00004400 \\ \mathrm{C} & 0.52558700 & 1.22852900 & 0.00038700 \\ \mathrm{C} & 1.90804400 & 1.11109000 & 0.00038100 \\ \mathrm{H} & -0.14012600 & -2.09470400 & -0.00057700 \\ \mathrm{H} & 0.05083600 & 2.19956200 & 0.00070100 \\ \mathrm{H} & 2.53186600 & 1.99653000 & 0.00065400 \\ \mathrm{C} & -1.67999000 & 0.09973500 & 0.00006000 \\ \mathrm{O} & -2.58082500 & -0.84888500 & 0.00024400 \\ \mathrm{~N} & -2.64573900 & 0.90232100 & -0.00048400 \\ \mathrm{~N} & 2.54514700 & -0.06125400 & 0.00000100 \\ \mathrm{C} & 1.81180600 & -1.17335700 & -0.00034000 \\ \mathrm{H} & 2.35717400 & -2.10923100 & -0.00062000\end{array}$

Zero-point correction $=$

0.089903

0.097014

0.097958

Thermal correction to Enthalpy=

0.057704

Thermal correction to Gibbs Free Energy=

Sum of electronic and zero-point Energies=

Sum of electronic and thermal Energies=

Sum of electronic and thermal Enthalpies=

Sum of electronic and thermal Free Energies=

$-415.696641$

$-415.689530$

$-415.688585$

$-415.728840$

\section{CBS-QB3}

$\begin{array}{lrrr}\mathrm{C} & -0.41896700 & -1.17305000 & 0.00038600 \\ \mathrm{C} & 0.23183200 & 0.05984700 & 0.00002500 \\ \mathrm{C} & -0.52572600 & 1.23254100 & -0.00038900 \\ \mathrm{C} & -1.91110200 & 1.11158000 & -0.00038600 \\ \mathrm{H} & 0.14629700 & -2.09689100 & 0.00069700 \\ \mathrm{H} & -0.05142100 & 2.20650900 & -0.00072600 \\ \mathrm{H} & -2.53854500 & 1.99759700 & -0.00072900 \\ \mathrm{C} & 1.68589000 & 0.09934000 & 0.00011500 \\ \mathrm{O} & 2.57766000 & -0.85854800 & -0.00070900 \\ \mathrm{~N} & 2.64786400 & 0.91228000 & 0.00075900 \\ \mathrm{~N} & -2.55097600 & -0.06299700 & -0.00002700 \\ \mathrm{C} & -1.81157600 & -1.17530100 & 0.00035600 \\ \mathrm{H} & -2.35793200 & -2.11355700 & 0.00066500\end{array}$

$\begin{array}{lclc}\text { Temperature }= & 298.150000 & \text { Pressure= } & 1.000000 \\ \text { E }(\text { ZPE })= & 0.088797 & \text { E(Thermal)= } & 0.096003 \\ \mathrm{E}(\text { SCF })= & -413.286289 & \text { DE(MP2)= } & -1.544225 \\ \text { DE(CBS })= & -0.145644 & \text { DE(MP34)= } & -0.030213 \\ \text { DE(CCSD })= & -0.064919 & \text { DE(Int)= } & 0.048437 \\ \text { DE(Empirical })= & -0.065461 & & \\ \text { CBS-QB3 }(0 \text { K })= & -414.999517 & \text { CBS-QB3 Energy= } & -414.992311 \\ \text { CBS-QB3 Enthalpy }= & -414.991367 & \text { CBS-QB3 Free Energy }= & -415.031854\end{array}$

$\begin{array}{lrrr}\text { isonicotinoyl nitrene-triplet (6) } & & \\ \text { B3LYP/6-311++G(3df,3pd) } & & \\ \mathrm{C} & 0.42102300 & -1.19434000 & 0.00005300 \\ \mathrm{C} & -0.28197500 & 0.00785500 & -0.00000300 \\ \mathrm{C} & 0.43602700 & 1.20190100 & -0.00005600 \\ \mathrm{C} & 1.82491100 & 1.13706700 & -0.00004300 \\ \mathrm{H} & -0.10878200 & -2.13576500 & 0.00009600 \\ \mathrm{H} & -0.06713100 & 2.15840600 & -0.00010300 \\ \mathrm{H} & 2.41379500 & 2.04645800 & -0.00009500\end{array}$




$\begin{array}{lrrr}\mathrm{C} & -1.77022700 & -0.00635600 & -0.00001100 \\ \mathrm{O} & -2.44986900 & -1.03219200 & -0.00003900 \\ \mathrm{~N} & -2.45464800 & 1.18693600 & 0.00005000 \\ \mathrm{~N} & 2.50481200 & -0.00790300 & 0.00000500 \\ \mathrm{C} & 1.80747800 & -1.14656200 & 0.00004800 \\ \mathrm{H} & 2.38649200 & -2.06218800 & 0.00010300\end{array}$

Zero-point correction $=$

0.089164

0.096207

0.097151

Thermal correction to Energy=

Thermal correction to Enthalpy=

0.055902

Sum of electronic and zero-point Energies=

$-415.703547$

$-415.696504$

$-415.695560$

Sum of electronic and thermal Enthalpies=

Sum of electronic and thermal Free Energies=

$-415.736809$

\section{CBS-QB3}

C

C

C

C

$\mathrm{H}$

$\mathrm{H}$

$\mathrm{H}$

C

$\mathrm{O}$

$\mathrm{N}$

$\mathrm{N}$

C

$\mathrm{H}$

Temperature $=$

$\mathrm{E}(\mathrm{ZPE})=$

$\mathrm{E}(\mathrm{SCF})=$

$\mathrm{DE}(\mathrm{CBS})=$

$\mathrm{DE}(\mathrm{CCSD})=$

$\mathrm{DE}($ Empirical $)=$

CBS-QB3 $(0 \mathrm{~K})=$

CBS-QB3 Enthalpy=

$\begin{array}{rrr}-0.41859300 & -1.19533000 & -0.00004900 \\ 0.28357900 & 0.01105400 & 0.00000100 \\ -0.43829000 & 1.20654800 & 0.00005100 \\ -1.82989600 & 1.13705600 & 0.00003300 \\ 0.11670500 & -2.13640900 & -0.00009200 \\ 0.06445800 & 2.16605400 & 0.00009800 \\ -2.42305800 & 2.04667400 & 0.00008800 \\ 1.77449500 & -0.00506900 & 0.00000600 \\ 2.45123800 & -1.03628200 & 0.00000000 \\ 2.46069700 & 1.18942800 & -0.00000100 \\ -2.51196800 & -0.01072900 & -0.00000300 \\ -1.80823300 & -1.14914900 & -0.00003700 \\ -2.38748400 & -2.06761900 & -0.00009200\end{array}$

$298.150000 \quad$ Pressure $=$

$0.088188 \quad \mathrm{E}($ Thermal $)=$

$-413.367805 \quad \mathrm{DE}(\mathrm{MP} 2)=$

$-0.140268 \quad \mathrm{DE}(\mathrm{MP} 34)=$

$-0.066265 \quad \mathrm{DE}(\mathrm{Int})=$

$-0.064914$

-414.994761 CBS-QB3 Energy=

-414.986738 CBS-QB3 Free Energy=
1.000000

0.095267

$-1.434468$

$-0.053302$

0.044074

$-414.987682$

$-415.027961$

\section{4-pyridyl isocyanate (7)}

\section{B3LYP/6-311++G(3df,3pd)}

$\begin{array}{lrrr}\mathrm{C} & -0.87185500 & -1.27089900 & 0.00000300 \\ \mathrm{C} & 0.08758500 & -0.26262900 & -0.00000400 \\ \mathrm{C} & -0.34467800 & 1.06373600 & -0.00001600 \\ \mathrm{C} & -1.70973500 & 1.31062200 & -0.00004400 \\ \mathrm{H} & -0.57283900 & -2.30870500 & 0.00002300 \\ \mathrm{H} & 0.36376600 & 1.88037500 & 0.00000000 \\ \mathrm{H} & -2.07061200 & 2.33264600 & -0.00001600 \\ \mathrm{~N} & 1.43359600 & -0.61017900 & 0.00001500 \\ \mathrm{C} & 2.51547300 & -0.08328200 & 0.00002800 \\ \mathrm{O} & 3.61341500 & 0.30696100 & 0.00002300 \\ \mathrm{C} & -2.21133300 & -0.90737500 & -0.00000700 \\ \mathrm{H} & -2.97698400 & -1.67434600 & 0.00002300 \\ \mathrm{~N} & -2.63979500 & 0.35493700 & -0.00001200\end{array}$


Zero-point correction $=$

0.092019

Thermal correction to Energy=

0.098957

Thermal correction to Enthalpy=

0.099901

Thermal correction to Gibbs Free Energy=

0.059663

Sum of electronic and zero-point Energies=

$-415.823745$

$-415.816807$

$-415.815863$

Sum of electronic and thermal Enthalpies=

Sum of electronic and thermal Free Energies=

$-415.856101$

\section{CBS-QB3}

$\begin{array}{lrrr}\mathrm{C} & -0.87260100 & -1.27384600 & -0.00000200 \\ \mathrm{C} & 0.08897700 & -0.26309400 & -0.00002900 \\ \mathrm{C} & -0.34454100 & 1.06638900 & -0.00003700 \\ \mathrm{C} & -1.71325800 & 1.31195300 & -0.00003000 \\ \mathrm{H} & -0.57116200 & -2.31329400 & 0.00006500 \\ \mathrm{H} & 0.36487800 & 1.88541700 & -0.00006000 \\ \mathrm{H} & -2.07637500 & 2.33579300 & 0.00004100 \\ \mathrm{~N} & 1.43755400 & -0.61411300 & -0.00001400 \\ \mathrm{C} & 2.52010800 & -0.08286900 & 0.00004200 \\ \mathrm{O} & 3.61895500 & 0.30940300 & 0.00006100 \\ \mathrm{C} & -2.21491800 & -0.90842700 & -0.00001600 \\ \mathrm{H} & -2.98305100 & -1.67637500 & 0.00000500 \\ \mathrm{~N} & -2.64734300 & 0.35591300 & -0.00000100\end{array}$

Temperature $=$

$\mathrm{E}(\mathrm{ZPE})=$

$\mathrm{E}(\mathrm{SCF})=$

$\mathrm{DE}(\mathrm{CBS})=$

$\mathrm{DE}(\mathrm{CCSD})=$

$\mathrm{DE}($ Empirical $)=$

CBS-QB3 $(0 \mathrm{~K})=$

CBS-QB3 Enthalpy=
298.150000

0.090980

$-413.430862$

$-0.145055$

$-0.059047$

$-0.066286$

$-415.115631$

$-415.107680$
Pressure $=$

$\mathrm{E}($ Thermal $)=$

$\mathrm{DE}(\mathrm{MP} 2)=$

$\mathrm{DE}(\mathrm{MP} 34)=$

$\mathrm{DE}($ Int $)=$

CBS-QB3 Energy=

CBS-QB3 Free Energy=
1.000000

0.097987

$-1.531397$

$-0.021990$

0.048025

$-415.108625$

$-415.148053$

\section{4-pyridyl nitrene-singlet (8)}

B3LYP/6-311++G(3df,3pd)

$\begin{array}{lrrr}\mathrm{C} & -1.18908300 & -1.03868900 & 0.00017800 \\ \mathrm{C} & 0.16468400 & -1.27818600 & 0.00012300 \\ \mathrm{C} & 1.07294100 & -0.18250300 & -0.00006900 \\ \mathrm{C} & 0.46197900 & 1.16490700 & -0.00017300 \\ \mathrm{C} & -0.91424300 & 1.19479000 & -0.00002900 \\ \mathrm{~N} & -1.75836800 & 0.16963200 & 0.00019100 \\ \mathrm{H} & -1.86438600 & -1.88723900 & 0.00047500 \\ \mathrm{H} & 0.57554400 & -2.27397800 & 0.00025100 \\ \mathrm{H} & 1.06989100 & 2.05148700 & -0.00033900 \\ \mathrm{H} & -1.34996300 & 2.19206200 & -0.00008500 \\ \mathrm{~N} & 2.32854700 & -0.06166800 & -0.00026000\end{array}$

Zero-point correction $=$

0.078097

Thermal correction to Energy=

0.083559

Thermal correction to Enthalpy=

0.084503

Thermal correction to Gibbs Free Energy=

Sum of electronic and zero-point Energies=

0.049049

Sum of electronic and thermal Energies=

$-302.299574$

$-302.294112$

$-302.293168$

Sum of electronic and thermal Enthalpies=

$-302.328622$ 


$\begin{array}{lrrc}\text { CBS-QB3 } & & & \\ \mathrm{C} & -1.18350400 & -1.04616500 & 0.00033600 \\ \mathrm{C} & 0.17493100 & -1.27721500 & 0.00014200 \\ \mathrm{C} & 1.07734500 & -0.17020100 & -0.00008700 \\ \mathrm{C} & 0.45342800 & 1.17033300 & -0.00016600 \\ \mathrm{C} & -0.92687800 & 1.19138800 & -0.00006900 \\ \mathrm{~N} & -1.76680200 & 0.15910500 & 0.00016400 \\ \mathrm{H} & -1.85412400 & -1.90161800 & 0.00038800 \\ \mathrm{H} & 0.59395200 & -2.27198100 & 0.00013800 \\ \mathrm{H} & 1.05654100 & 2.06318500 & -0.00033500 \\ \mathrm{H} & -1.37327700 & 2.18645600 & -0.00018000 \\ \mathrm{~N} & 2.33894200 & -0.05694400 & -0.00029900\end{array}$

$\begin{array}{lclc}\text { Temperature }= & 298.150000 & \text { Pressure= } & 1.000000 \\ \mathrm{E}(\mathrm{ZPE})= & 0.077125 & \mathrm{E}(\text { Thermal })= & 0.082647 \\ \mathrm{E}(\mathrm{SCF})= & -300.501155 & \mathrm{DE}(\mathrm{MP})= & -1.122014 \\ \mathrm{DE}(\mathrm{CBS})= & -0.106192 & \mathrm{DE}(\mathrm{MP3})= & -0.041434 \\ \mathrm{DE}(\mathrm{CCSD})= & -0.052755 & \mathrm{DE}(\mathrm{Int})= & 0.036213 \\ \text { DE(Empirical })= & -0.049809 & & \\ \text { CBS-QB3 }(0 \mathrm{~K})= & -301.760020 & \text { CBS-QB3 Energy= } & -301.754498 \\ \text { CBS-QB3 Enthalpy }= & -301.753554 & \text { CBS-QB3 Free Energy= } & -301.789116\end{array}$

\section{4-pyridyl nitrene-triplet (8)}

\section{B3LYP/6-311++G(3df,3pd)}

$\begin{array}{lrrr}\mathrm{C} & 1.06532900 & 1.14630800 & 0.00028400 \\ \mathrm{C} & -0.31249000 & 1.21527300 & 0.00013000 \\ \mathrm{C} & -1.05625600 & 0.00000000 & -0.00009200 \\ \mathrm{C} & -0.31248900 & -1.21527300 & -0.00020600 \\ \mathrm{C} & 1.06533000 & -1.14630700 & -0.00004900 \\ \mathrm{~N} & 1.75896300 & 0.00000000 & 0.00019700 \\ \mathrm{H} & 1.65283500 & 2.05687900 & 0.00045400 \\ \mathrm{H} & -0.83180100 & 2.16221400 & 0.00018700 \\ \mathrm{H} & -0.83180000 & -2.16221500 & -0.00040400 \\ \mathrm{H} & 1.65283600 & -2.05687900 & -0.00011000 \\ \mathrm{~N} & -2.37876500 & 0.00000000 & -0.00027300\end{array}$

Zero-point correction $=$

0.079178

Thermal correction to Energy=

0.084343

0.085287

Thermal correction to Enthalpy=

0.049397

$-302.361090$

$-302.355925$

$-302.354981$

$-302.390871$

\section{CBS-QB3}

$\begin{array}{lrrr}\mathrm{C} & 1.06813900 & 1.14716500 & 0.00026100 \\ \mathrm{C} & -0.31335200 & 1.21713100 & 0.00013700 \\ \mathrm{C} & -1.05994900 & -0.00000100 & -0.00015000 \\ \mathrm{C} & -0.31335200 & -1.21713100 & -0.00019500 \\ \mathrm{C} & 1.06814000 & -1.14716400 & -0.00002200 \\ \mathrm{~N} & 1.76599000 & 0.00000000 & 0.00019100 \\ \mathrm{H} & 1.65762400 & 2.05938800 & 0.00048200 \\ \mathrm{H} & -0.83400000 & 2.16602400 & 0.00023800 \\ \mathrm{H} & -0.83400000 & -2.16602500 & -0.00037500 \\ \mathrm{H} & 1.65762400 & -2.05938800 & -0.00009100\end{array}$


Temperature $=$

$\mathrm{E}(\mathrm{ZPE})=$

$\mathrm{E}(\mathrm{SCF})=$

$\mathrm{DE}(\mathrm{CBS})=$

$\mathrm{DE}(\mathrm{CCSD})=$

$\mathrm{DE}($ Empirical $)=$

CBS-QB3 $(0 \mathrm{~K})=$

CBS-QB3 Enthalpy=
298.150000

$$
0.078258
$$

$-300.623345$

$-0.103179$

$-0.058528$

$-0.052181$

$-301.820998$

$-301.814839$
Pressure=

$\mathrm{E}($ Thermal $)=$

$\mathrm{DE}(\mathrm{MP} 2)=$

$\mathrm{DE}(\mathrm{MP} 34)=$

$\mathrm{DE}(\operatorname{Int})=$

CBS-QB3 Energy=

CBS-QB3 Free Energy=
1.000000

0.083472

$-1.028982$

$-0.065080$

0.032038

$-301.815784$

$-301.850812$

TS1: anti-syn nicotinoyl azide (1) $\rightarrow$ anti 3-pyridyl isocyanate (3)

\section{CBS-QB3}

$\begin{array}{lrrr}\mathrm{C} & -1.16160400 & -1.12904000 & 0.34708200 \\ \mathrm{C} & -0.52308600 & 0.09016400 & 0.10657600 \\ \mathrm{C} & -1.27533600 & 1.16612300 & -0.36751800 \\ \mathrm{C} & -2.63970000 & 0.98358100 & -0.54170000 \\ \mathrm{H} & -0.58982700 & -1.98443300 & 0.69290100 \\ \mathrm{H} & -0.80221300 & 2.11841700 & -0.57645100 \\ \mathrm{H} & -3.27153800 & 1.78852100 & -0.89725900 \\ \mathrm{C} & 0.96192800 & 0.30935400 & 0.50798900 \\ \mathrm{O} & 1.34469800 & 0.89760700 & 1.49600000 \\ \mathrm{~N} & 1.47484900 & -0.29339900 & -0.54098900 \\ \mathrm{~N} & 3.21098600 & -0.21152600 & -0.32831100 \\ \mathrm{~N} & 4.23159400 & -0.40280900 & -0.69003800 \\ \mathrm{C} & -3.18666700 & -0.26899300 & -0.25685900 \\ \mathrm{H} & -4.24988400 & -0.44547100 & -0.39263800 \\ \mathrm{~N} & -2.47211500 & -1.31093900 & 0.17773600\end{array}$

Temperature $=$

$\mathrm{E}(\mathrm{ZPE})=$

$\mathrm{E}(\mathrm{SCF})=$

$\mathrm{DE}(\mathrm{CBS})=$

$\mathrm{DE}(\mathrm{CCSD})=$

$\mathrm{DE}($ Empirical $)=$

CBS-QB3 $(0 \mathrm{~K})=$

CBS-QB3 Enthalpy=

$\begin{array}{cc}298.150000 & \text { Pressure }= \\ 0.095869 & \mathrm{E}(\text { Thermal })= \\ -522.258189 & \text { DE(MP2)= } \\ -0.179057 & \text { DE(MP34)= } \\ -0.076257 & \text { DE(Int) }= \\ -0.081229 & \end{array}$

$-524.378283$

$-524.367697$
CBS-QB3 Energy=

CBS-QB3 Free Energy=
1.000000

0.105511

$-1.905722$

$-0.033122$

0.059425

$-524.368641$

$-524.415240$

TS2: anti-syn nicotinoyl azide (1-I) $\rightarrow$ anti-anti nicotinoyl azide (1-II)

\section{CBS-QB3}

$\begin{array}{lrcc}\mathrm{C} & 0.67589700 & -0.99197000 & -0.47754700 \\ \mathrm{C} & 0.38305800 & 0.33377000 & -0.13554100 \\ \mathrm{C} & 1.42301200 & 1.13824900 & 0.34650300 \\ \mathrm{C} & 2.68494500 & 0.58278500 & 0.48041600 \\ \mathrm{H} & -0.09750400 & -1.63518200 & -0.88365200 \\ \mathrm{H} & 1.21921400 & 2.17029700 & 0.60462400 \\ \mathrm{H} & 3.51834900 & 1.16514200 & 0.85389000 \\ \mathrm{C} & -0.97051300 & 0.92813800 & -0.27839400 \\ \mathrm{O} & -1.21204400 & 2.09502100 & -0.14817600 \\ \mathrm{~N} & -2.02352100 & 0.00244000 & -0.69285900 \\ \mathrm{~N} & -2.57879000 & -0.66995100 & 0.17392300 \\ \mathrm{~N} & -3.16276300 & -1.32528200 & 0.88892200 \\ \mathrm{C} & 2.86412900 & -0.75474600 & 0.12183700 \\ \mathrm{H} & 3.83984000 & -1.22237800 & 0.21892200 \\ \mathrm{~N} & 1.88840100 & -1.53370800 & -0.35333300\end{array}$




$\begin{array}{lclc}\text { Temperature }= & 298.150000 & \text { Pressure= } & 1.000000 \\ \mathrm{E}(\text { ZPE })= & 0.100097 & \mathrm{E}(\text { Thermal })= & 0.108154 \\ \mathrm{E}(\mathrm{SCF})= & -522.273553 & \mathrm{DE}(\mathrm{MP})= & -1.940809 \\ \mathrm{DE}(\mathrm{CBS})= & -0.181928 & \mathrm{DE}(\mathrm{MP34})= & -0.016781 \\ \mathrm{DE}(\mathrm{CCSD})= & -0.075232 & \mathrm{DE}(\mathrm{Int})= & 0.059898 \\ \mathrm{DE}(\text { Empirical })= & -0.081264 & & \\ \text { CBS-QB3 }(0 \mathrm{~K})= & -524.409573 & \text { CBS-QB3 Energy }= & -524.401516 \\ \text { CBS-QB3 Enthalpy }= & -524.400572 & \text { CBS-QB3 Free Energy }= & -524.443645\end{array}$

\begin{tabular}{|c|c|c|c|c|c|}
\hline \multicolumn{6}{|l|}{ CBS-QB3 } \\
\hline $\mathrm{C}$ & 0.54803700 & \multicolumn{2}{|c|}{-0.93708900} & -0.52270200 & \\
\hline $\mathrm{C}$ & 0.35184700 & \multicolumn{2}{|c|}{0.37010500} & -0.06568200 & \\
\hline $\mathrm{C}$ & 1.45761700 & \multicolumn{2}{|c|}{1.06350900} & 0.43587100 & \\
\hline $\mathrm{C}$ & 2.68218800 & \multicolumn{2}{|c|}{0.41485400} & 0.49655600 & \\
\hline $\mathrm{H}$ & -0.26683100 & \multicolumn{2}{|c|}{-1.49593400} & -0.97006400 & \\
\hline $\mathrm{H}$ & 1.33647300 & \multicolumn{2}{|c|}{2.08866600} & 0.76464700 & \\
\hline $\mathrm{H}$ & 3.56099600 & \multicolumn{2}{|c|}{0.91205300} & 0.88859000 & \\
\hline $\mathrm{C}$ & -0.92932300 & \multicolumn{2}{|c|}{1.11542100} & -0.14133900 & \\
\hline $\mathrm{O}$ & -0.99827200 & \multicolumn{2}{|c|}{2.35942200} & -0.21933000 & \\
\hline $\mathrm{N}$ & -2.19566800 & \multicolumn{2}{|c|}{0.72432800} & -0.14442500 & \\
\hline $\mathrm{N}$ & -2.39178800 & \multicolumn{2}{|c|}{-1.02895400} & 0.17571200 & \\
\hline $\mathrm{N}$ & -3.11739300 & \multicolumn{2}{|c|}{-1.82211000} & 0.41442700 & \\
\hline $\mathrm{C}$ & 2.76120000 & \multicolumn{2}{|c|}{-0.89865800} & 0.03540700 & \\
\hline $\mathrm{H}$ & 3.70255100 & \multicolumn{2}{|c|}{-1.43937800} & 0.07155600 & \\
\hline $\mathrm{N}$ & 1.72278600 & \multicolumn{2}{|c|}{-1.56571900} & -0.47689900 & \\
\hline Temperature $=$ & \multicolumn{2}{|c|}{298.150000} & \multicolumn{2}{|c|}{$\begin{array}{l}\text { Pressure }= \\
\mathrm{E}(\text { Thermal) }=\end{array}$} & 1.000000 \\
\hline $\mathrm{E}(\mathrm{ZPE})=$ & \multicolumn{2}{|c|}{0.096256} & $\mathrm{E}(1$ & hermal) $=$ & 0.105649 \\
\hline $\mathrm{E}(\mathrm{SCF})=$ & \multicolumn{2}{|c|}{-522.244326} & \multicolumn{2}{|c|}{$\mathrm{DE}(\mathrm{MP} 2)=$} & -1.903902 \\
\hline $\mathrm{DE}(\mathrm{CBS})=$ & \multicolumn{2}{|c|}{-0.178900} & \multicolumn{2}{|c|}{$\mathrm{DE}(\mathrm{MP} 34)=$} & -0.038762 \\
\hline $\mathrm{DE}(\mathrm{CCSD})=$ & \multicolumn{2}{|c|}{-0.078768} & & $(\operatorname{Int})=$ & 0.059598 \\
\hline DE(Empirical)= & \multicolumn{2}{|c|}{-0.081154} & \multirow{2}{*}{\multicolumn{2}{|c|}{ CBS-OB3 Energy= }} & \\
\hline CBS-QB3 $(0 \mathrm{~K})=$ & \multicolumn{2}{|c|}{-524.369958} & & & -524.360565 \\
\hline CBS-QB3 Enthalpy= & \multicolumn{2}{|c|}{-524.359621} & $\mathrm{CB}$ & -QB3 Free Energy= & -524.405930 \\
\hline
\end{tabular}

TS4: anti nicotinoyl nitrene-singlet (2) $\rightarrow$ anti 3-pyridyl isocyanate (3) CBS-QB3

$\begin{array}{lrrc}\mathrm{C} & 0.43080900 & -1.17819900 & -0.16853400 \\ \mathrm{C} & -0.29072600 & 0.01768100 & -0.00750800 \\ \mathrm{C} & 0.41547500 & 1.21806300 & 0.17208500 \\ \mathrm{C} & 1.79665700 & 1.16977300 & 0.13272600 \\ \mathrm{H} & -0.09935600 & -2.11630700 & -0.29682200 \\ \mathrm{H} & -0.12073300 & 2.14908100 & 0.31308700 \\ \mathrm{H} & 2.39501900 & 2.06569700 & 0.24388400 \\ \mathrm{C} & -1.78758900 & 0.00439900 & -0.01051600 \\ \mathrm{O} & -2.59462400 & 0.53637100 & -0.79780500 \\ \mathrm{C} & 2.41863800 & -0.07422700 & -0.04093700 \\ \mathrm{H} & 3.50277200 & -0.14148800 & -0.05468700 \\ \mathrm{~N} & 1.76223200 & -1.22807100 & -0.18851500 \\ \mathrm{~N} & -2.15889200 & -0.64750400 & 1.03068600\end{array}$

Temperature $=$ $\mathrm{E}(\mathrm{ZPE})=$
$298.150000 \quad$ Pressure $=$ $0.087252 \quad \mathrm{E}($ Thermal $)=$
1.000000

0.094231 


$\begin{array}{lclc}\mathrm{E}(\mathrm{SCF})= & -413.287861 & \mathrm{DE}(\mathrm{MP} 2)= & -1.497598 \\ \mathrm{DE}(\mathrm{CBS})= & -0.143306 & \mathrm{DE}(\mathrm{MP3})= & -0.041720 \\ \mathrm{DE}(\mathrm{CCSD})= & -0.066674 & \mathrm{DE}(\mathrm{Int})= & 0.047658 \\ \mathrm{DE}(\text { Empirical })= & -0.065946 & & \\ \text { CBS-QB3 }(0 \mathrm{~K})= & -414.968195 & \text { CBS-QB3 Energy= } & -414.961216 \\ \text { CBS-QB3 Enthalpy }= & -414.960272 & \text { CBS-QB3 Free Energy }= & -415.000534\end{array}$

TS1: syn isonicotinoyl azide (5) $\rightarrow$ 4-pyridyl isocyanate (7) CBS-QB3

$\begin{array}{lrrr}\mathrm{C} & -1.21299600 & -1.20168300 & -0.01195100 \\ \mathrm{C} & -0.52057900 & -0.00057600 & 0.12065200 \\ \mathrm{C} & -1.21246500 & 1.20179900 & -0.00282500 \\ \mathrm{C} & -2.58736500 & 1.14380900 & -0.21904600 \\ \mathrm{H} & -0.70245300 & -2.15412800 & 0.05753200 \\ \mathrm{H} & -0.70149500 & 2.15346200 & 0.07383800 \\ \mathrm{H} & -3.16383000 & 2.05948900 & -0.31333900 \\ \mathrm{C} & 0.97221500 & -0.00277700 & 0.58584500 \\ \mathrm{O} & 1.33487800 & -0.00825100 & 1.74103600 \\ \mathrm{~N} & 1.48860200 & 0.00284200 & -0.61804300 \\ \mathrm{~N} & 3.22683800 & 0.00175900 & -0.38315100 \\ \mathrm{~N} & 4.24991000 & 0.00351700 & -0.78560200 \\ \mathrm{~N} & -3.27131300 & 0.00175400 & -0.33354100 \\ \mathrm{C} & -2.58786000 & -1.14145100 & -0.22770000 \\ \mathrm{H} & -3.16473600 & -2.05613200 & -0.32889600\end{array}$

$\begin{array}{lclc}\text { Temperature }= & 298.150000 & \text { Pressure= } & 1.000000 \\ \text { E }(\text { ZPE })= & 0.095721 & \text { E(Thermal)= } & 0.105390 \\ \text { E(SCF })= & -522.255646 & \text { DE(MP2)= } & -1.907102 \\ \text { DE(CBS })= & -0.179317 & \text { DE(MP34)= } & -0.032735 \\ \text { DE }(\text { CCSD })= & -0.076360 & \text { DE(Int })= & 0.059544 \\ \text { DE(Empirical })= & -0.081189 & & \\ \text { CBS-QB3 }(0 \text { K })= & -524.377082 & \text { CBS-QB3 Energy= } & -524.367413 \\ \text { CBS-QB3 Enthalpy }= & -524.366469 & \text { CBS-QB3 Free Energy }= & -524.414297\end{array}$

TS2: syn isonicotinoyl azide (5-I) $\rightarrow$ anti isonicotinoyl azide (5-II)

\section{CBS-QB3}

$\begin{array}{lrrr}\mathrm{C} & 1.41413300 & 1.12633000 & 0.34510900 \\ \mathrm{C} & 0.37970500 & 0.32357800 & -0.14420300 \\ \mathrm{C} & 0.66879600 & -0.99831100 & -0.48554600 \\ \mathrm{C} & 1.97430500 & -1.45565200 & -0.31533100 \\ \mathrm{H} & 1.22113400 & 2.15783600 & 0.61020400 \\ \mathrm{H} & -0.09295800 & -1.65138600 & -0.89066100 \\ \mathrm{H} & 2.23277700 & -2.47766600 & -0.57593600 \\ \mathrm{C} & -0.98070100 & 0.92179600 & -0.28704200 \\ \mathrm{O} & -1.21759400 & 2.08851000 & -0.16155700 \\ \mathrm{~N} & -2.03247400 & -0.00544100 & -0.69464100 \\ \mathrm{~N} & -2.59465300 & -0.66859600 & 0.17470900 \\ \mathrm{~N} & -3.18402800 & -1.31696400 & 0.89143900 \\ \mathrm{~N} & 2.96668800 & -0.70022900 & 0.16016600 \\ \mathrm{C} & 2.67931000 & 0.56664000 & 0.48066100 \\ \mathrm{H} & 3.50342100 & 1.16110300 & 0.86332200\end{array}$

Temperature $=$

$\mathrm{E}(\mathrm{ZPE})=$

$\mathrm{E}(\mathrm{SCF})=$

$$
\begin{array}{cl}
298.150000 & \text { Pressure }= \\
0.100012 & \mathrm{E}(\text { Thermal })= \\
-522.269743 & \text { DE }(\text { MP2 })=
\end{array}
$$

\author{
1.000000 \\ 0.108062
}

$-1.944319$ 
DE $(C B S)=$
DE(CCSD $)=$
DE(Empirical)=
CBS-QB3 $(0 \mathrm{~K})=$
CBS-QB3 Enthalpy=

$$
\begin{gathered}
-0.182586 \\
-0.075156 \\
-0.081174 \\
-524.408335 \\
-524.399341
\end{gathered}
$$

$\mathrm{DE}(\mathrm{MP} 34)=$

$\mathrm{DE}(\mathrm{Int})=$

CBS-QB3 Energy=

CBS-QB3 Free Energy=
$-0.015480$

0.060112

$-524.400285$

$-524.442436$

TS3: anti isonicotinoyl azide (5-II) $\rightarrow$ isonicotinoyl nitrene-singlet (6)

\section{CBS-QB3}

$\begin{array}{lrrr}\mathrm{C} & 0.54750300 & -0.91322500 & -0.58559900 \\ \mathrm{C} & 0.33712200 & 0.36993200 & -0.08002200 \\ \mathrm{C} & 1.42292900 & 1.04730700 & 0.47731300 \\ \mathrm{C} & 2.65381800 & 0.40067300 & 0.53392000 \\ \mathrm{H} & -0.24357200 & -1.47165800 & -1.06795300 \\ \mathrm{H} & 1.29871100 & 2.05487000 & 0.85359300 \\ \mathrm{H} & 3.51250200 & 0.89643900 & 0.97672000 \\ \mathrm{C} & -0.95189500 & 1.11305900 & -0.15090300 \\ \mathrm{O} & -1.02619900 & 2.35506400 & -0.23522700 \\ \mathrm{~N} & -2.21216600 & 0.70835200 & -0.14040700 \\ \mathrm{~N} & -2.37527700 & -1.05307000 & 0.18423500 \\ \mathrm{~N} & -3.08326900 & -1.85963400 & 0.42996900 \\ \mathrm{~N} & 2.86375200 & -0.83185000 & 0.06178400 \\ \mathrm{C} & 1.82516900 & -1.46099900 & -0.49346000 \\ \mathrm{H} & 2.02180400 & -2.45346800 & -0.88839700\end{array}$

Temperature $=$

$\mathrm{E}(\mathrm{ZPE})=$

$\mathrm{E}(\mathrm{SCF})=$

$\mathrm{DE}(\mathrm{CBS})=$

$\mathrm{DE}(\mathrm{CCSD})=$

$\mathrm{DE}($ Empirical $)=$

CBS-QB3 $(0 \mathrm{~K})=$

CBS-QB3 Enthalpy=

$\begin{array}{cl}298.150000 & \text { Pressure }= \\ 0.096140 & \text { E (Thermal })= \\ -522.241332 & \text { DE }(\text { MP2 })= \\ -0.179352 & \text { DE(MP34) }= \\ -0.078612 & \text { DE(Int) }= \\ -0.081074 & \\ -524.369052 & \text { CBS-QB3 Energy }= \\ -524.358701 & \text { CBS-QB3 Free Energy }=\end{array}$

1.000000

0.105547

$-1.907046$

$-0.037527$

0.059752

$-524.359645$

$-524.405049$

$\begin{array}{lrcc}\text { TS4: isonicotinoyl nitrene-singlet }(6) \rightarrow \text { 4-pyridyl isocyanate (7) } \\ \text { CBS-QB3 } & & & \\ \text { C } & 0.43307900 & -1.19114000 & -0.19959300 \\ \text { C } & -0.29540000 & -0.01480800 & 0.00697500 \\ \text { C } & 0.39081500 & 1.18795000 & 0.22043900 \\ \text { C } & 1.77784300 & 1.15874200 & 0.16563100 \\ \text { H } & -0.07028100 & -2.13717800 & -0.35148400 \\ \text { H } & -0.14694600 & 2.11148400 & 0.39477200 \\ \text { H } & 2.35170300 & 2.07061400 & 0.29908200 \\ \text { C } & -1.80469600 & -0.01602300 & -0.01703300 \\ \text { O } & -2.57991900 & 0.56427000 & -0.79898100 \\ \text { N } & -2.18691700 & -0.71189200 & 0.99020900 \\ \text { N } & 2.48883800 & 0.03983500 & -0.03610800 \\ \text { C } & 1.82528500 & -1.10225100 & -0.21804700 \\ \text { H } & 2.42987400 & -1.98949900 & -0.37945900\end{array}$

Temperature $=$

$\mathrm{E}(\mathrm{ZPE})=$

$\mathrm{E}(\mathrm{SCF})=$

$\mathrm{DE}(\mathrm{CBS})=$

$\mathrm{DE}(\mathrm{CCSD})=$

$\mathrm{DE}($ Empirical $)=$
298.150000 Pressure $=$
$0.086868 \quad \mathrm{E}($ Thermal $)=$
$-413.284201 \quad$ DE(MP2) $=$
-0.143888 DE(MP34) $=$
$-0.065490 \quad \mathrm{DE}(\mathrm{Int})=$
$-0.065899$

1.000000
0.093873
-1.499046
-0.040916
0.047866 


$\begin{array}{lllc}\text { CBS-QB3 }(0 \mathrm{~K})= & -414.964707 & \text { CBS-QB3 Energy }= & -414.957702 \\ \text { CBS-QB3 Enthalpy }= & -414.956758 & \text { CBS-QB3 Free Energy }= & -414.997179\end{array}$

CASSCF(12,11)/cc-pVTZ calculated energies (in Hartrees) of nicotinoyl nitrene (2) and isonicotinoyl nitrene (6)

nicotinoyl nitrene (2)

anti-closed shell singlet (CSS)

\begin{tabular}{|c|c|}
\hline 20222220000 & 0.8839353 \\
\hline 02222220000 & -0.2294800 \\
\hline 20222020200 & -0.1348938 \\
\hline 20222202000 & -0.1101193 \\
\hline 20 222baab00 & -0.0772996 \\
\hline 20 222abba00 & -0.0772996 \\
\hline 20 222bbaa00 & 0.0629149 \\
\hline 20 222aabb00 & 0.0629149 \\
\hline $\mathrm{ab} 22 \mathrm{~b} 2200 \mathrm{a} 0$ & -0.0580446 \\
\hline ba $22 \mathrm{a} 2200 \mathrm{~b} 0$ & -0.0580446 \\
\hline 20 2b2a20a0b & -0.0549402 \\
\hline $202 \mathrm{a} 2 \mathrm{~b} 20 \mathrm{~b} 0 \mathrm{a}$ & -0.0549402 \\
\hline 20220220020 & -0.0545464 \\
\hline ba 22a22b000 & -0.0504682 \\
\hline ab 22b22a000 & -0.0504682 \\
\hline
\end{tabular}

1.2863429026

C $\quad-0.0132970380$

$-0.2726815255 \quad 0.0000000000$

$0.2339056024 \quad 0.0000000000$

$-0.6530466660 \quad 0.0000000000$

$-2.0114055592 \quad 0.0000000000$

$\begin{array}{ll}0.3929533869 & 0.0000000000\end{array}$

$\begin{array}{ll}-0.2907783427 & 0.0000000000\end{array}$

$\begin{array}{ll}-2.7397578616 & 0.0000000000\end{array}$

$\begin{array}{ll}1.6724221921 & 0.0000000000\end{array}$

$2.3093669026 \quad 0.0000000000$

$\begin{array}{ll}-2.4166873856 & 0.0000000000\end{array}$

$\begin{array}{ll}-3.4604851406 & 0.0000000000\end{array}$

$\begin{array}{ll}-1.5692041186 & 0.0000000000\end{array}$

$\begin{array}{ll}2.7310725158 & 0.0000000000\end{array}$

ENERGIES

$-413.47640625$

anti-open shell singlet (OSS)

\begin{tabular}{|c|c|}
\hline 2b $2222 \mathrm{a} 0000$ & 0.6470724 \\
\hline 2a $2222 \mathrm{~b} 0000$ & -0.6470724 \\
\hline $2 \mathrm{~b} 2202 \mathrm{a} 0200$ & -0.0998087 \\
\hline 2a $2202 b 0200$ & 0.0998087 \\
\hline 2b $2220 \mathrm{a} 2000$ & -0.0832736 \\
\hline 2a $2220 b 2000$ & 0.0832736 \\
\hline 2b 2b2aaa0b0 & 0.0531232 \\
\hline 2a $2 \mathrm{a} 2 \mathrm{bbb} 0 \mathrm{a} 0$ & -0.0531232 \\
\hline
\end{tabular}




$\begin{array}{lr}\text { 2b 22baaab00 } & -0.0514803 \\ \text { 2a 22abbba00 } & 0.0514803 \\ \text { 2b 2a2bab0a0 } & 0.0504731 \\ \text { 2a 2b2aba0b0 } & -0.0504731 \\ \text { 2b 22ababa00 } & -0.0502901 \\ \text { 2a 22babab00 } & 0.0502901\end{array}$

$\begin{array}{lrrr}\mathrm{C} & -1.1970073899 & -0.4848070861 & 0.0000000000 \\ \mathrm{C} & -0.0244628416 & 0.2786612947 & 0.0000000000 \\ \mathrm{C} & 1.1960905770 & -0.3879664196 & 0.0000000000 \\ \mathrm{C} & 1.1895335586 & -1.7770344810 & 0.0000000000 \\ \mathrm{H} & -2.1563165341 & -0.0041408331 & 0.0000000000 \\ \mathrm{H} & 2.1158896384 & 0.1602794591 & 0.0000000000 \\ \mathrm{H} & 2.1060069721 & -2.3328606713 & 0.0000000000 \\ \mathrm{C} & -0.0815985208 & 1.7585188232 & 0.0000000000 \\ \mathrm{O} & 1.0716056171 & 2.4438653527 & 0.0000000000 \\ \mathrm{C} & -0.0305596514 & -2.4390771062 & 0.0000000000 \\ \mathrm{H} & -0.0704783244 & -3.5116444068 & 0.0000000000 \\ \mathrm{~N} & -1.2016344337 & -1.8072426170 & 0.0000000000 \\ \mathrm{~N} & -1.1560676672 & 2.4334516914 & 0.0000000000\end{array}$

ENERGIES

$-413.43564357$

\section{anti-triplet}

\begin{tabular}{|c|c|c|c|}
\hline 2a $2222 \mathrm{a} 0000$ & \multicolumn{2}{|c|}{0.9161235} & \\
\hline 2a $2202 \mathrm{a} 0200$ & 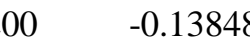 & & \\
\hline 2a $2220 \mathrm{a} 2000$ & -0.11163 & & \\
\hline 2a 22baaab00 & -0.07851 & & \\
\hline 2a 22ababa00 & -0.07447 & & \\
\hline 2a 2022a0020 & -0.06483 & & \\
\hline $2 \mathrm{a} 2 \mathrm{~b} 2 \mathrm{aaa} 0 \mathrm{~b} 0$ & -0.06396 & & \\
\hline 2a $2 \mathrm{a} 2 \mathrm{bab} 0 \mathrm{a} 0$ & -0.06068 & & \\
\hline 2a 22aaabb00 & 0.0597 & & \\
\hline 2a 22 bbaaa00 & 0.0592 & & \\
\hline 2a 2022a2000 & -0.05790 & & \\
\hline \multirow{2}{*}{$\begin{array}{l}2 \mathrm{a} a 2 \mathrm{~b} 2 \mathrm{a} 0 \mathrm{~b} 0 \mathrm{a} \\
2 \mathrm{a} b 2 \mathrm{a} 2 \mathrm{a} 0 \mathrm{a} 0 \mathrm{~b}\end{array}$} & 0.0565 & & \\
\hline & 0.0564 & & \\
\hline $\begin{array}{l}\text { 2a b2a2a0a0b } \\
2 \mathrm{a} 2 \mathrm{a} 22 \mathrm{~b} 00 \mathrm{a} 0\end{array}$ & $0.0549^{\prime}$ & & \\
\hline \multicolumn{4}{|c|}{$2 \mathrm{a} 2 \mathrm{~b} 2 \mathrm{aab0a0} \quad 0.0501524$} \\
\hline $\mathrm{C}$ & -1.1756163557 & -0.4636180438 & 0.0000000000 \\
\hline $\mathrm{C}$ & 0.0028758584 & 0.2793341009 & 0.0000000000 \\
\hline $\mathrm{C}$ & 1.2196124726 & -0.4039767811 & 0.0000000000 \\
\hline $\mathrm{C}$ & 1.1956942657 & -1.7868608051 & 0.0000000000 \\
\hline $\mathrm{H}$ & -2.1311852859 & 0.0240311995 & 0.0000000000 \\
\hline $\mathrm{H}$ & \multirow{2}{*}{2.1417936066} & 0.1413016317 & 0.0000000000 \\
\hline $\mathrm{H}$ & & -2.3577887171 & 0.0000000000 \\
\hline $\mathrm{C}$ & $\begin{array}{r}2.1028540757 \\
-00132009326\end{array}$ & 1.7635887620 & 0.0000000000 \\
\hline $\mathrm{O}$ & 0.9868568490 & 2.4424134876 & 0.0000000000 \\
\hline $\mathrm{C}$ & -0.0392876184 & -2.4341621742 & 0.0000000000 \\
\hline $\mathrm{H}$ & -0.0903423022 & -3.5065692999 & 0.0000000000 \\
\hline $\mathrm{N}$ & -1.1982952712 & -1.7922216126 & 0.0000000000 \\
\hline $\mathrm{N}$ & -1.2407383621 & 2.4245312522 & 0.0000000000 \\
\hline \multicolumn{2}{|c|}{ ENERGIES } & -413.4788104 & \\
\hline
\end{tabular}


syn-closed shell singlet (CSS)

\begin{tabular}{|c|c|c|c|}
\hline 20222220000 & \multicolumn{2}{|c|}{0.8836609} & \\
\hline 02222220000 & -0.23068 & & \\
\hline 20222020200 & -0.0967 & & \\
\hline 20222202000 & -0.0822 & & \\
\hline 20222200200 & -0.0680 & & \\
\hline $20222 \mathrm{aabb00}$ & 0.0627 & & \\
\hline 20222 bbaa00 & 0.0627 & & \\
\hline $\mathrm{ab} 22 \mathrm{~b} 2200 \mathrm{a} 0$ & 0.0581 & & \\
\hline ba $22 \mathrm{a} 2200 \mathrm{~b} 0$ & 0.05811 & & \\
\hline 20222022000 & -0.0556 & & \\
\hline 20220220020 & $-0.0540^{\prime}$ & & \\
\hline ba $22 \mathrm{a} 22 \mathrm{~b} 000$ & -0.05035 & & \\
\hline $\mathrm{ab} 22 \mathrm{~b} 22 \mathrm{a} 000$ & -0.05035 & & \\
\hline \multicolumn{4}{|c|}{20222 baab00 } \\
\hline \multicolumn{4}{|c|}{$20222 \mathrm{abba00} \quad-0.0502210$} \\
\hline $\mathrm{C}$ & -0.9829355932 & -0.7550250371 & 0.0000000000 \\
\hline $\mathrm{C}$ & -0.0079284268 & 0.2378880800 & 0.0000000000 \\
\hline $\mathrm{C}$ & 1.3355095967 & -0.1326396951 & 0.0000000000 \\
\hline $\mathrm{C}$ & 1.6361651539 & -1.4832363445 & 0.0000000000 \\
\hline $\mathrm{H}$ & -2.0236247953 & -0.4934681301 & 0.0000000000 \\
\hline $\mathrm{H}$ & 2.1089643283 & 0.6106971399 & 0.0000000000 \\
\hline $\mathrm{H}$ & 2.6515586538 & -1.8257791993 & 0.0000000000 \\
\hline $\mathrm{C}$ & -0.3995524500 & 1.6416137402 & 0.0000000000 \\
\hline $\mathrm{O}$ & -1.5792638791 & 2.1616257122 & 0.0000000000 \\
\hline $\mathrm{C}$ & 0.5865590703 & -2.4010172727 & 0.0000000000 \\
\hline $\mathrm{H}$ & 0.7890694332 & -3.4551650041 & 0.0000000000 \\
\hline $\mathrm{N}$ & -0.6922340429 & -2.0501174429 & 0.0000000000 \\
\hline $\mathrm{N}$ & 0.1591219511 & 2.7627484534 & 0.0000000000 \\
\hline
\end{tabular}

ENERGIES

$-413.47628011$

syn-open shell singlet (OSS)

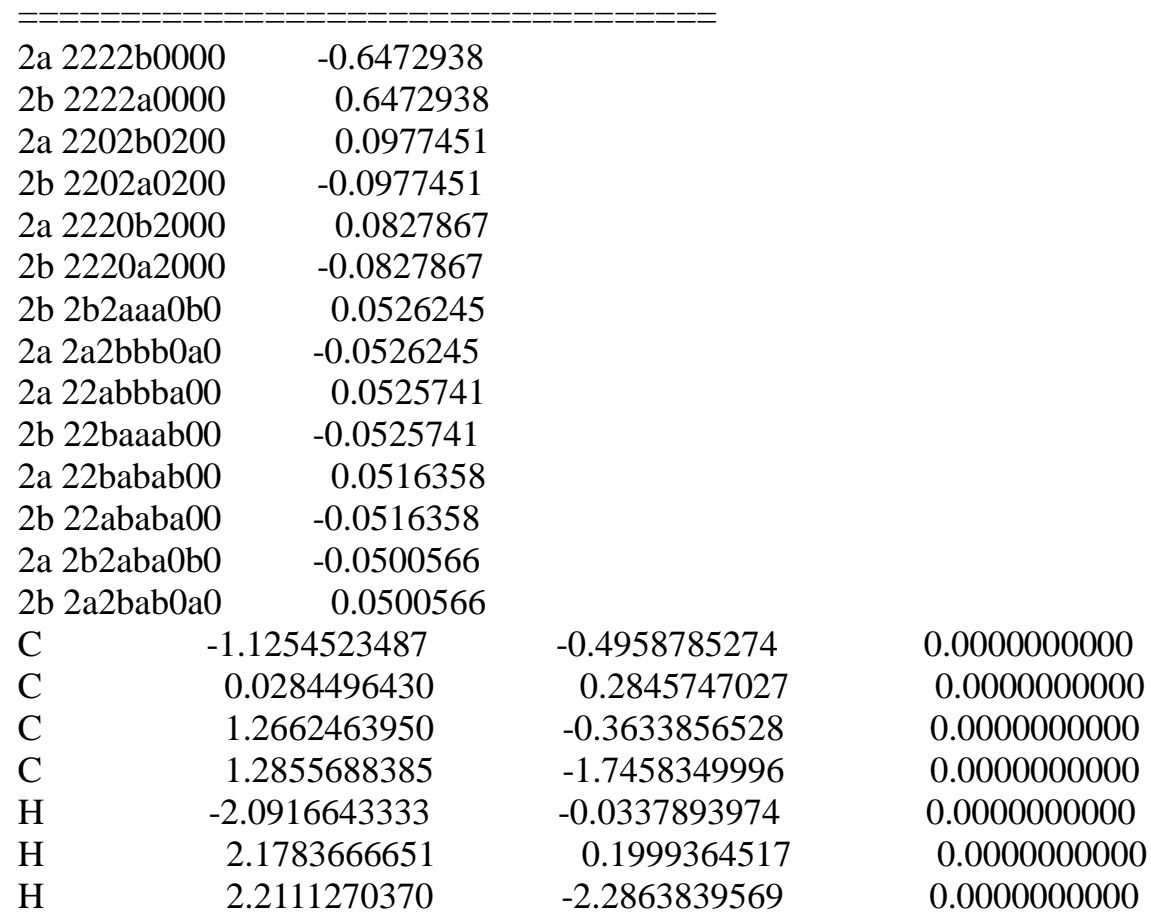




$\begin{array}{lrrr}\mathrm{C} & -0.0670737971 & 1.7617911095 & 0.0000000000 \\ \mathrm{O} & -1.2818512679 & 2.3318349351 & 0.0000000000 \\ \mathrm{C} & 0.0718789015 & -2.4324430094 & 0.0000000000 \\ \mathrm{H} & 0.0545549010 & -3.5059058463 & 0.0000000000 \\ \mathrm{~N} & -1.1048231237 & -1.8255179706 & 0.0000000000 \\ \mathrm{~N} & 0.9376044894 & 2.5366821612 & 0.0000000000\end{array}$

ENERGIES

$-413.43566176$

\section{syn-triplet}

\begin{tabular}{|c|c|c|c|}
\hline 2a 2222a0000 & \multicolumn{2}{|c|}{0.9156165} & \\
\hline \multirow{2}{*}{$\begin{array}{l}\text { 2a 2202a0200 } \\
\text { 2a 2220a2000 }\end{array}$} & -0.1379 & & \\
\hline & -0.1115 & & \\
\hline $\begin{array}{l}\text { 2a 2220a2000 } \\
\text { 2a } 22 \text { baaab00 }\end{array}$ & 0.0754 & & \\
\hline 2a 22ababa00 & 0.0710 & & \\
\hline 2a 2022a0020 & -0.0655 & & \\
\hline $2 \mathrm{a} 2 \mathrm{~b} 2 \mathrm{aaa} 0 \mathrm{~b} 0$ & 0.0646 & & \\
\hline 2a 2a2bab0a0 & 0.0613 & & \\
\hline 2a 22aaabb00 & $-0.0599 x$ & & \\
\hline 2a 22bbaaa00 & -0.0594 & & \\
\hline 2a $2022 \mathrm{a} 2000$ & -0.0575 & & \\
\hline $2 \mathrm{a} a 2 \mathrm{~b} 2 \mathrm{a} 0 \mathrm{~b} 0 \mathrm{a}$ & 0.0559 & & \\
\hline 2a b2a2a0a0b & 0.0558 & & \\
\hline $2 \mathrm{a} 2 \mathrm{a} 22 \mathrm{~b} 00 \mathrm{a} 0$ & 0.0554 & & \\
\hline \multirow{2}{*}{$\begin{array}{l}\text { 2a } 2 b 2 a a b 0 a 0 \\
\text { 2a } 2220 a 0020\end{array}$} & -0.0512 & & \\
\hline & -0.0510 & & \\
\hline $\begin{array}{ll}\mathrm{C} & -1.1 \\
\mathrm{C} & 0 .\end{array}$ & 1.1469717523 & -0.5179291468 & 0.0000000000 \\
\hline \multirow{2}{*}{$\begin{array}{l}\mathrm{C} \\
\mathrm{C}\end{array}$} & 0.0008168835 & 0.2798589692 & 0.0000000000 \\
\hline & 1.2431076779 & -0.3477960002 & 0.0000000000 \\
\hline C & 1.2810188175 & -1.7355001051 & 0.0000000000 \\
\hline \multirow{2}{*}{$\begin{array}{r}-2 . \\
2 .\end{array}$} & 2.1158226421 & -0.0596819201 & 0.0000000000 \\
\hline & 2.1506292398 & 0.2229876238 & 0.0000000000 \\
\hline $\mathrm{H}$ & 2.2137764127 & -2.2633407725 & 0.0000000000 \\
\hline \multirow{2}{*}{$\begin{array}{l}\mathrm{C} \\
\mathrm{O}\end{array}$} & 0.1356083910 & 1.7579987680 & 0.0000000000 \\
\hline & 1.1959995371 & 2.3378855924 & 0.0000000000 \\
\hline $\mathrm{C}$ & 0.0803864123 & -2.4342225008 & 0.0000000000 \\
\hline \multirow[t]{2}{*}{$\mathrm{H}$} & 0.0751610316 & -3.5076434069 & 0.0000000000 \\
\hline & 1.1100792312 & -1.8411949349 & 0.0000000000 \\
\hline $\mathrm{N}$ & 1.0225170783 & 2.5342578338 & 0.0000000000 \\
\hline
\end{tabular}

ENERGIES

$-413.47832352$

isonicotinoyl nitrene (6)

closed shell singlet (CSS)

$\begin{array}{lr}=====================0.8815292 \\ 20222220000 & -0.2314881 \\ 02222220000 & -0.1364897 \\ 20222020200 & -0.1185516 \\ 20222202000 & 0.0800163 \\ 20222 \mathrm{baab} 00 & 0.0800163 \\ 20222 \mathrm{abba00} & -0.0651840 \\ 20222 \mathrm{bbaa} 00 & -0.0651840\end{array}$




$\begin{array}{lr}\text { ab 22b2200a0 } & -0.0610026 \\ \text { ba 22a2200b0 } & -0.0610026 \\ 20220220020 & -0.0591030 \\ 202 \mathrm{~b} 2 \mathrm{a} 20 \mathrm{a} 0 \mathrm{~b} & 0.0536353 \\ 202 \mathrm{a} 2 \mathrm{~b} 20 \mathrm{~b} 0 \mathrm{a} & 0.0536353 \\ 202 \mathrm{a} 22 \mathrm{bb} 00 \mathrm{a} & -0.0516387 \\ 202 \mathrm{~b} 22 \mathrm{aa} 00 \mathrm{~b} & -0.0516387 \\ \text { ba 22a22b000 } & -0.0507237 \\ \text { ab 22b22a000 } & -0.0507237\end{array}$

$\begin{array}{lccc}\mathrm{C} & -1.0363838035 & -0.6924711815 & 0.0000000000 \\ \mathrm{C} & -0.0111027336 & 0.2440125840 & 0.0000000000 \\ \mathrm{C} & 1.3079409021 & -0.2007021853 & 0.0000000000 \\ \mathrm{C} & 1.5367199903 & -1.5698120994 & 0.0000000000 \\ \mathrm{H} & -2.0644709964 & -0.3898158393 & 0.0000000000 \\ \mathrm{H} & 2.1281438194 & 0.4895612192 & 0.0000000000 \\ \mathrm{H} & 2.5395709640 & -1.9513478660 & 0.0000000000 \\ \mathrm{C} & -0.3204151635 & 1.6715672824 & 0.0000000000 \\ \mathrm{O} & -1.4706723557 & 2.2533982560 & 0.0000000000 \\ \mathrm{~N} & 0.3028210742 & 2.7574459092 & 0.0000000000 \\ \mathrm{~N} & 0.5609461443 & -2.4732076080 & 0.0000000000 \\ \mathrm{C} & -0.6930333050 & -2.0411169373 & 0.0000000000 \\ \mathrm{H} & -1.4579875367 & -2.7936975339 & 0.0000000000\end{array}$

ENERGIES

$-413.47568391$

open shell singlet (OSS)

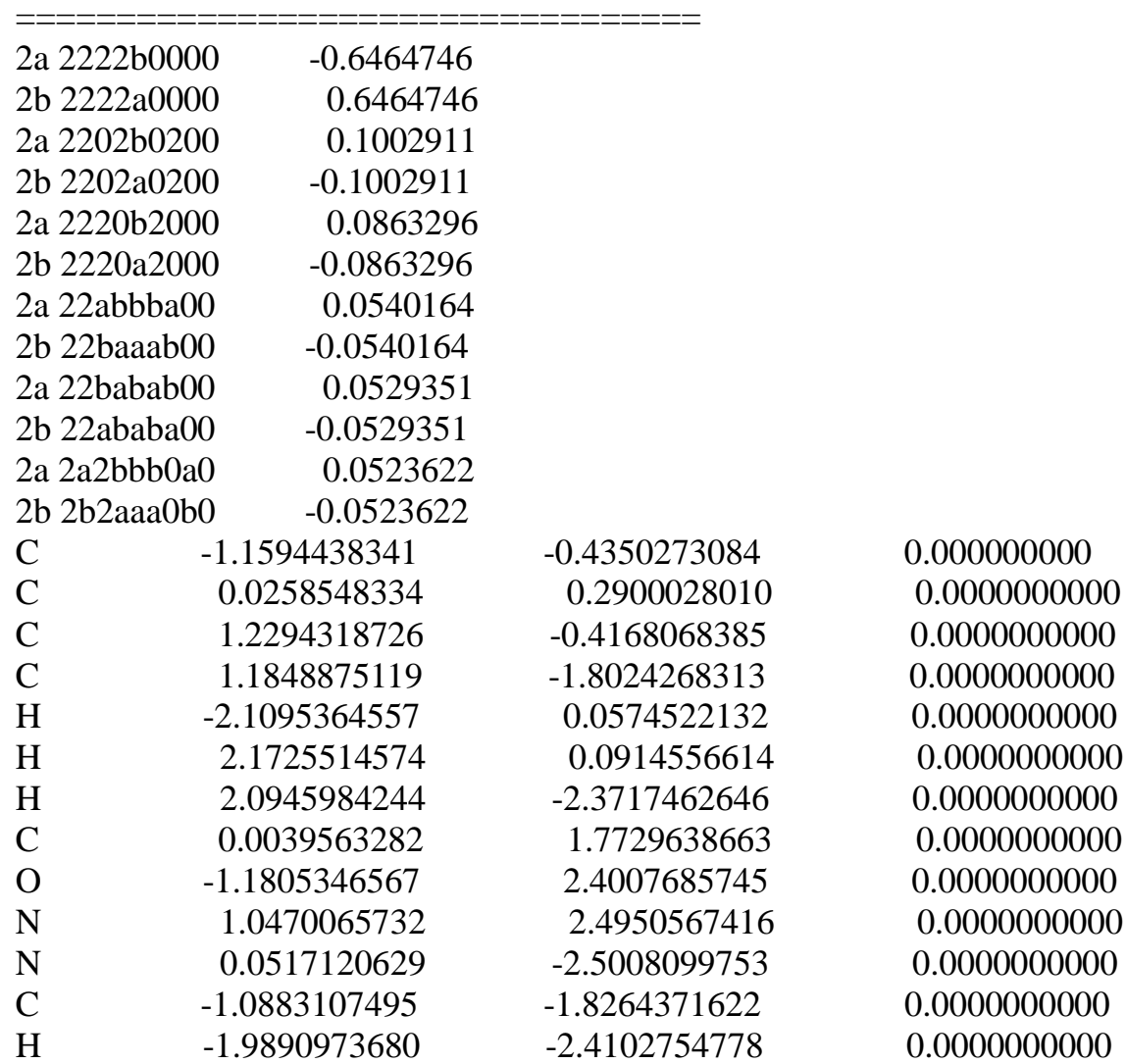




\section{triplet}

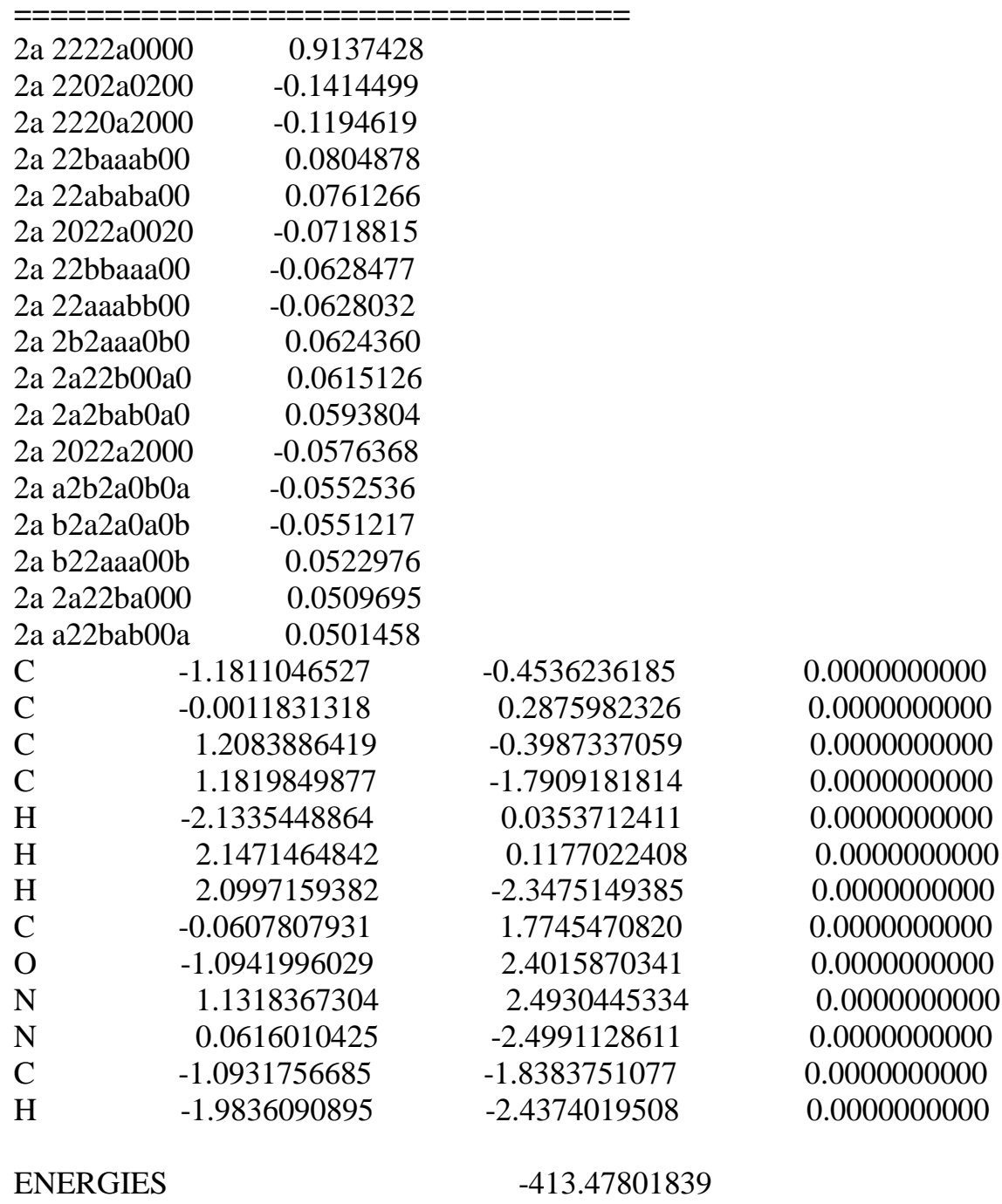

